\title{
High Resolution Laser Spectroscopy of Cesium Vapor Layers with Nanometric Thickness
}

\author{
Stefka Cartaleva ${ }^{1}$, Anna Krasteva ${ }^{1}$, Armen Sargsyan ${ }^{2}$, \\ David Sarkisyan², Dimitar Slavovi, Petko Todorov ${ }^{1}$ and Kapka Vaseva1 \\ ${ }^{1}$ Institute of Electronics, Bulgarian Academy of Sciences, Sofia \\ 2Institute for Physical Research, National Academy of Sciences of Armenia, Ashtarak \\ ${ }^{1}$ Bulgaria \\ ${ }^{2}$ Armenia
}

\section{Introduction}

High resolution laser spectroscopy of alkali vapor contained in conventional thermal optical cells with centimeter dimensions is widely used for various applications: among them wavelength references, atomic clocks, precise optical magnetometers, slow and stored light etc. For all these photonic sensors, the reduction of their dimensions is of significant importance. One of the main concerns is to keep the parameters of the photonic sensor when reducing its size. In this chapter are presented the obtained by authors experimental and theoretical results concerning high-resolution spectroscopy of Cs vapor layer with nanometric thickness. The thickness of the vapor layer varies from $100 \mathrm{~nm}$ to about $5000 \mathrm{~nm}$. The practical importance of this study is accompanied by numerous new peculiarities of atomic spectra of $1 \mathrm{D}$ confined atoms, when the nanometric dimension approaches the wavelength of the irradiating light. These peculiarities in the absorption and fluorescence spectra represent a basic importance as well.

\section{Unique optical cells for confinement of Cs atomic layers with nanometric thickness}

\subsection{Main characteristics of atomic confinement}

In this chapter, the high resolution laser spectroscopy is concerned of alkali vapor confined in unique optical cell with nanometric thickness [Sarkisyan, 2001], further on called Extremely Thin Cell (ETC). The transversal and longitudinal dimensions of such cell (Fig.1) differ significantly. The distance between the high-quality ETC windows L varies from $100 \mathrm{~nm}$ to (1-3) $\mu \mathrm{m}$. At the same time, the cell window diameter is about $2 \mathrm{~cm}$ (Fig.1b). Therefore, a strong spatial anisotropy is present for the time of interaction between atoms confined in the ETC and the laser radiation used for spectroscopy performed with such optical cell.

Let us consider Cs atoms flying orthogonally to the cell windows (Fig.1a, atoms denoted by $\mathrm{v} \perp$ ), which average thermal velocity at room temperature is about $200 \mathrm{~m} / \mathrm{s}$. Those atoms 
will pass the $\mathrm{L}=1 \mu \mathrm{m}$ distance for $5 \mathrm{~ns}$. Hence the time of flight of atoms is much shorter than the lifetime of the excited atomic state. Such a limit is not imposed on the atoms (Fig.1a, denoted by $\mathrm{v}_{\mathrm{II}}$ ), moving parallel to the windows of the ETC. The second group of atoms will interact with the laser radiation for a time determined by the diameter of the laser beam $D$ (D >> L). When the ETC is irradiated by a laser beam propagating in direction orthogonal to the ETC window surfaces, the atoms with velocity direction close to parallel to the window surface can be considered as "slow" atoms, i.e. atoms with very small velocity projection on the laser beam direction. Hence two groups of atoms can be mainly distinguished - "slow" (moving parallel to the windows) and "fast" (moving parallel to the laser beam propagation direction, in the extremely narrow space between the two windows of the ETC). As a first result of the light interaction with those atomic groups, a strong reduction of the Doppler effect influence occurs and of the related Doppler broadening of spectral lines as well.

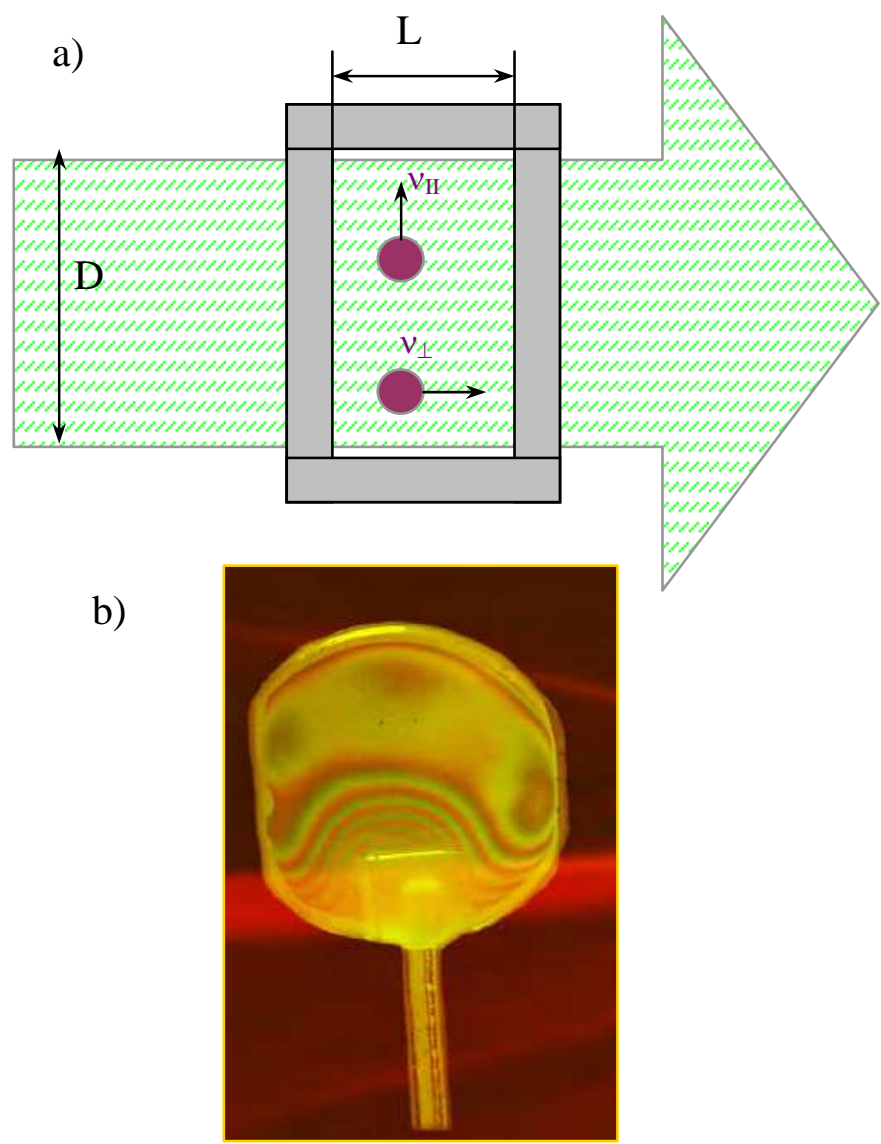

Fig. 1. (a) Atomic movement in cell of nanometric thickness L: $\mathrm{V}_{\text {II }}$ - velocity component parallel to the cell windows; $\mathrm{v} \perp$ - atomic velocity component orthogonal to the windows and along the laser beam, (b) Practical realization of nanometric cell. 


\subsection{Hyperfine transitions on the $D_{2}$ line of $C s$ atoms}

In this chapter, the spectral properties of Cs atoms confined in ETC are particularly concerned. The diagram of ${ }^{133} \mathrm{Cs}$ energy levels with the hyperfine transitions on the $\mathrm{D}_{2}$ line is shown in Fig.2.

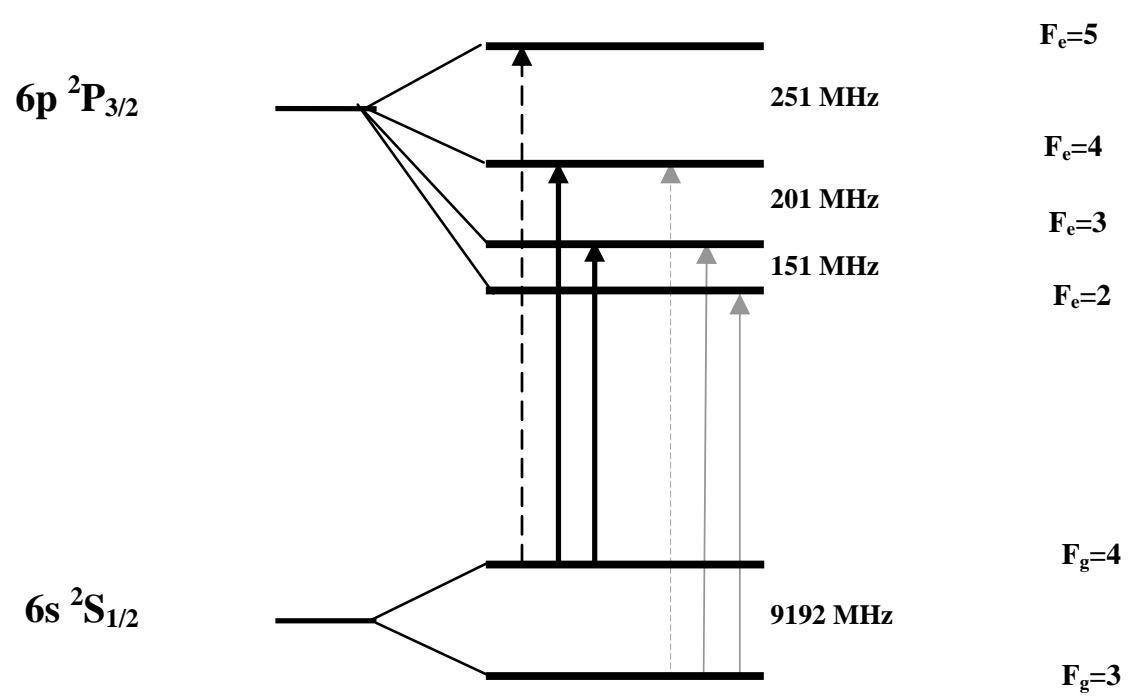

Fig. 2. Energy-level diagram for $D_{2}$ line of ${ }^{133}$ Cs. $F_{g} \rightarrow F_{e} \leq F_{g}$ transitions (solid line) are distinguished from $\mathrm{F}_{\mathrm{g}} \rightarrow \mathrm{F}_{\mathrm{e}}>\mathrm{F}_{\mathrm{g}}$ transitions (dashed line). The wavelength of the $\mathrm{D}_{2}$ line is $\lambda=852 \mathrm{~nm}$.

Cesium $\mathrm{D}_{2}$ line consists of two sets of hyperfine transitions, forming two absorption (fluorescence) lines:

- $\quad \mathrm{F}_{\mathrm{g}}=3$ set, involving three hyperfine transitions starting from the $\mathrm{F}_{\mathrm{g}}=3$ ground level to the respective $\mathrm{F}_{\mathrm{e}}=2,3,4$ excited levels and

- $\mathrm{F}_{\mathrm{g}}=4$ set of transitions - the $\mathrm{F}_{\mathrm{g}}=4 \rightarrow \mathrm{F}_{\mathrm{e}}=3,4,5$ hyperfine transitions.

In the widely used optical cells with centimeter dimensions (further on called conventional cells), the hyperfine transitions starting from single ground level completely overlap due to the Doppler broadening (the spectral width $\sim 400 \mathrm{MHz}$, for individual hyperfine transition), which is larger than the separation between the excited state hyperfine levels. Thus, the two types of transitions $\left(\mathrm{F}_{\mathrm{g}} \rightarrow \mathrm{F}_{\mathrm{e}} \leq \mathrm{F}_{\mathrm{g}}\right.$ or $\left.\mathrm{F}_{\mathrm{g}} \rightarrow \mathrm{F}_{\mathrm{e}}>\mathrm{F}_{\mathrm{g}}\right)$ involved in the absorption line can not be resolved. However, they have different properties. The first type $\left(F_{g} \rightarrow F_{e} \leq F_{g}\right.$ transition) can suffer population loss from the excited by the light ground level due to hyperfine optical pumping to the other ground hyperfine level, which does not interact with the light. When this type of transition is closed for hyperfine optical pumping, it intrinsically will exhibit Zeeman optical pumping to ground-state Zeeman sublevels non-interacting with the light. In opposite, if the transition of the second type $\left(F_{g} \rightarrow F_{e}>F_{g}\right.$ transition) is closed for hyperfine optical pumping, it can be considered as a completely closed one [Andreeva, 2007, 
a; Andreeva, 2007, b; Andreeva, 2002], i.e. the atomic population is cycling only between the energy levels involved in the atomic transition, with all Zeeman sublevels of the ground level excited by the light.

The situation is different in the case of ETC. Here the hyperfine transitions within a single absorption (fluorescence) line can be resolved [Andreeva, 2007, a; Andreeva, 2007, b] (see Fig.3). Hence we can investigate separately the two types of hyperfine transitions, which are involved in the absorption (fluorescence) line by means of very simple single beam spectroscopy. The $F_{g}=4 \rightarrow F_{e}=3,4,5$ set of transitions involves the $F_{g}=4 \rightarrow F_{e}=5$ transition, which is the only completely closed one on the $\mathrm{D}_{2}$ line.

The presence of the two main groups of atoms, determined by the ETC anisotropy, leads also to the observation of a significant difference between the fluorescence and transmission spectra (Fig. 3). In the ETC, the basic contribution to the creation of fluorescence signal belongs to the atoms moving parallel to the windows, i.e. "slow" atoms. In fact, the "fast" atoms moving along the laser beam direction do not have enough time to perform a complete absorption-emission cycle, i.e. to fluoresce. Hence, strong narrowing of the fluorescence profiles occur and the hyperfine optical transitions are completely resolved in the fluorescence case.

The situation with the absorption is different. Here, together with the "slow" atoms, the "fast" atoms are also able to contribute to the absorption spectrum of the nanometric atomic vapor layer, because the absorption of a quantum of light is a process a lot faster than the fluorescence. In this way, the absorption profile "suffers" much more from the Doppler effect. Hence, because different velocity groups of atoms contribute to the fluorescence and absorption profile formation, a big difference in the width of the corresponding optical transitions occurs (Fig.3).

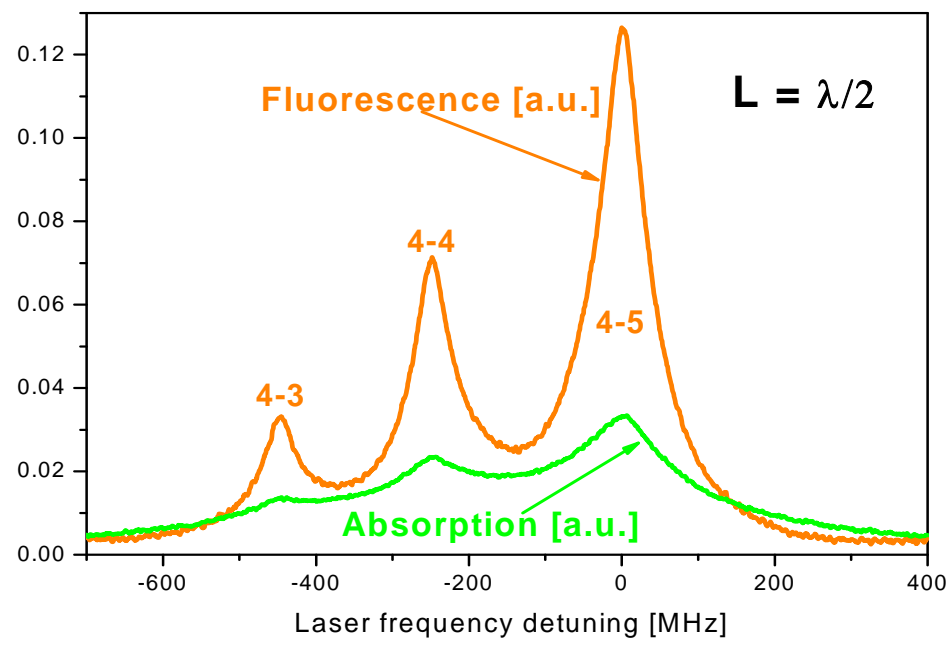

Fig. 3. Difference between the spectral widths of fluorescence and absorption profiles; the illustration is on the $\mathrm{D}_{2}$ line of $\mathrm{Cs}$, for optical transitions starting from the ground level $F_{g}=4$; the 4-3, 4-4 and 4-5 are the respective hyperfine transitions. $\lambda=852 \mathrm{~nm}$. 


\section{Main properties of the experimental fluorescence and absorption spectra of $\mathrm{Cs}$ atoms confined in nanometric cells}

As above mentioned, in the conventional thermal cells the different hyperfine Cs transitions starting from single ground level $\mathrm{F}_{\mathrm{g}}$ are completely overlapped and cannot be resolved at all. Consequently, the application of the nanometric cell provides a new opportunity for significant enhancement of the resolution in laser spectroscopy of thermal cell, without application of complex atomic beam or laser cooling systems. Moreover, this simple tool makes it possible to study also the dynamic processes in the absorption and fluorescence, as well as in the electromagnetically induced transparency and absorption.

\subsection{Sub-Doppler resonances in the absorption (transmission) of confined in nanometric cell atoms - Experimental observations}

\subsubsection{Experimental set up}

A schematic drawing of the experimental set up is presented in Fig.4. Three different radiation sources emitting at $\lambda=852 \mathrm{~nm}$ were used in the experiments presented here:

- a free running diode laser with linewidth of about $15 \mathrm{MHz}$,

- a cw Extended Cavity Diode Laser (ECDL) operating at single-frequency mode with linewidth of about $3 \mathrm{MHz}$, and

- a Distributed Feedback Laser (DFB) with very low-noise current controller and linewidth of about $2 \mathrm{MHz}$.

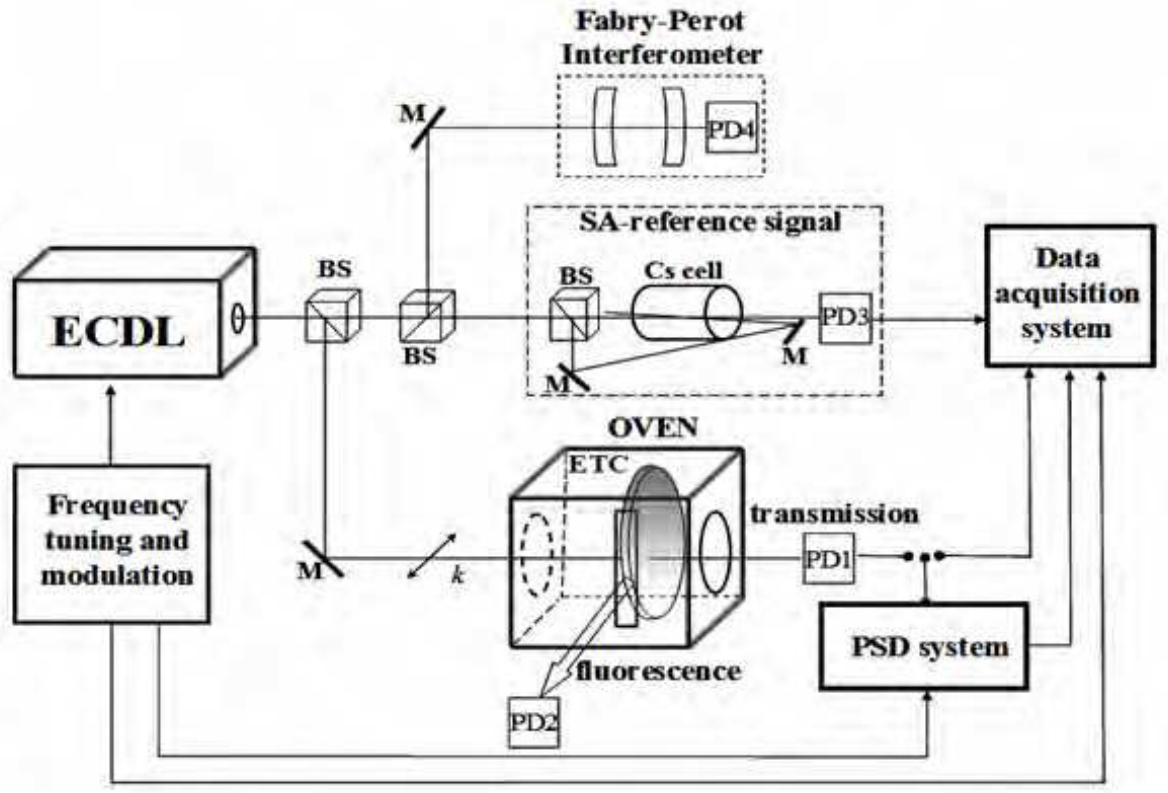

Fig. 4. Experimental set up 
For all laser systems, the laser light is linearly polarized and its frequency is scanned in the region of the hyperfine transitions starting from ground-state levels with quantum numbers $\mathrm{F}_{\mathrm{g}}=3$ and $\mathrm{F}_{\mathrm{g}}=4$, at the $\mathrm{D}_{2}$ line of $\mathrm{Cs}$ (see Fig.2).

The main part of the laser beam is directed at normal incidence onto the ETC filled with Cs vapor from a side-arm source. The construction of the ETC with a wedge-shaped (tunable) gap between high optical quality windows is similar to that reported in Ref. [Sarkisyan, 2001], but in some of the experiments the thickness of the vapor layer may vary in the range of $(350-5000) \mathrm{nm}$. This enhancement of the thickness variation range is achieved by a preceding deposition of about $5000 \mathrm{~nm}$ thick $\mathrm{Al}_{2} \mathrm{O}_{3}$ layer onto the surface of one of the ETC windows in its lower part. The wedge-shaped (along the vertical direction) thickness of the ETC was measured by the interference technique described in Ref. [Dutier, 2003, b]. The dimensions of the ETC windows are $20 \mathrm{~mm} \times 30 \mathrm{~mm} \times 2.5 \mathrm{~mm}$. A hole of $2 \mathrm{~mm}$ in diameter was drilled in the bottom of the windows, into which a tube of the same diameter and $50 \mathrm{~mm}$ long, made of commercial sapphire, was inserted. Then, the entire construction was assembled and glued in a vacuum furnace. After the gluing, a glass extension was sealed in the sapphire tube, and the ETC was filled with Cs metal, as it is usually done for conventional glass cells. The transmitted through the ETC beam is measured by a photodiode PD1 in dependence on the laser frequency, and the fluorescence is registered by a photodiode PD2.

The remaining part of the laser beam is used for laser frequency control:

- one beam is sent to a scanning Fabry-Perot interferometer for monitoring the singlemode operation of the ECDL;

- the second one - to an additional branch of the set-up including conventional, 3-cm long Cs cell with $2 \mathrm{~cm}$ diameter, for registration of the Saturated Absorption (SA) spectrum (PD-3).

The SA spectrum is recorded simultaneously with the signal from the ETC branch, thus ensuring precise frequency reference and scaling for the laser system frequency tuning. The sub-Doppler spectra in the transmitted beam and in fluorescence are recorded for different laser light intensities and ETC thicknesses.

\subsubsection{Absorption spectra of atoms confined in ETC with $L=\lambda / 2$ and $L=\lambda$}

For illustration of the narrowing of absorption spectra of atomic transitions in ETC and their strong dependence on the cell thickness, first the experimentally observed spectrum only at two cell thicknesses is discussed.

In Fig.5a, the absorption spectrum of $6 \mathrm{~S}_{1 / 2}\left(\mathrm{~F}_{\mathrm{g}}=4\right) \rightarrow 6 \mathrm{P}_{3 / 2}\left(\mathrm{~F}_{\mathrm{e}}=3,4,5\right)$ hyperfine transitions is presented, for three different irradiating light intensities and $L=\lambda / 2$. The strong narrowing of each hyperfine transition is clearly seen, which is due to the absorption enhancement in the transition center relative to that in the wings. The origin of the narrowing of the hyperfine transition profiles is attributed to the anisotropy of the atomlight interaction time and to the Dicke effect [Romer]. Processes responsible for the coherent Dicke narrowing of the hyperfine transition profile at $L=\lambda / 2$ can be briefly summarized as follows [Dutier, 2003, a; Sarkisyan, 2004; Maurin]. If an atom at the moment of leaving the cell wall is excited by resonant light, the excitation will start to precess in phase with the exciting 

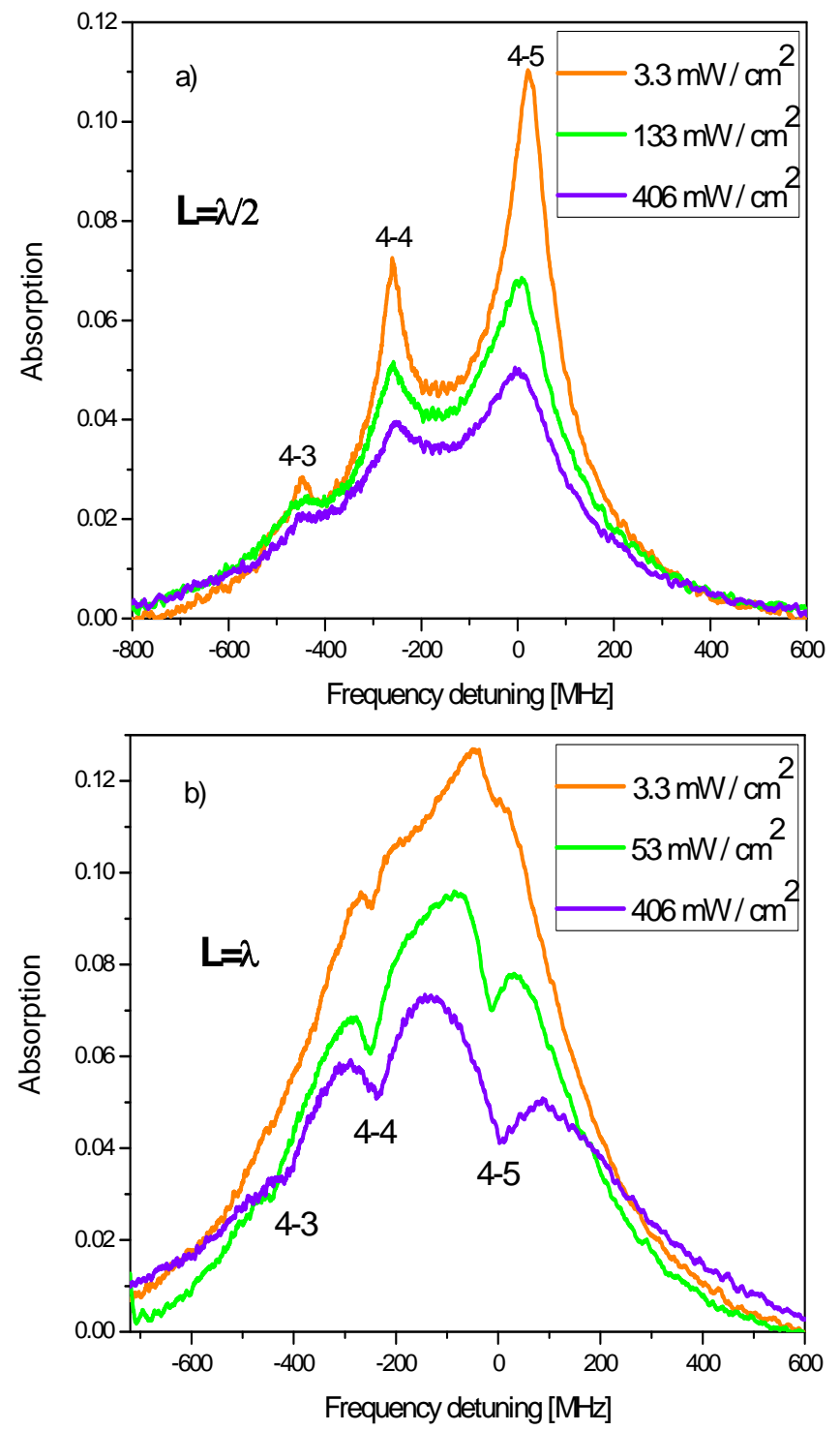

Fig. 5. Absorption spectra for the $\mathrm{F}_{\mathrm{g}}=4$ set of transitions, at three light intensities: (a) $L=\lambda / 2$ and (b) $L=\lambda$.

electromagnetic field at the wall position. However, with atomic motion the excitation will go gradually out of phase with the local exciting field. The phase mismatch appearing on the line center under a weak exciting field is independent of the atomic velocity and for a cell thickness up to $\lambda / 2$ all regions of the cell interfere constructively, leading to a strong absorption enhancement at the hyperfine transition center. However, if the exciting light is detuned from the hyperfine transition center, the angular precession of the atomic excitation 
becomes velocity-dependent resulting in smooth reduction of the absorption in the wings of the hyperfine profile. From Fig.5a, we can also see the power broadening of the observed sub-Doppler-width profiles, together with a reduction in the overall absorption with irradiation light power density.

In Fig.5b, the absorption spectra at $6 \mathrm{~S}_{1 / 2}\left(\mathrm{~F}_{\mathrm{g}}=4\right) \rightarrow 6 \mathrm{P}_{3 / 2}\left(\mathrm{~F}_{\mathrm{e}}=3,4,5\right)$ set of transitions are shown, for $L=\lambda$. One could immediately notice the significant difference observed between the absorption spectrum at $L=\lambda / 2$ and that at $L=\lambda$. For $L=\lambda$, the coherent Dicke narrowing vanishes and as a result of the Doppler broadening the hyperfine structure is not resolved at very low intensity. However, rising the light intensity, it is possible to observe at cell thickness equal to $\lambda$ well pronounced narrow dips of velocity selective reduced absorption, centered at the hyperfine transitions. The origin of these dips is related to the fact that processes like the two-level atomic system saturation and optical pumping can be completed only for atoms with large enough time of interaction with the laser light, i.e. "slow" atoms. Thus, the atoms flying nearly parallel to the ETC windows undergo optical pumping process (for the open transitions) and a saturation process (for all transitions) and this gives rise to highly velocityselective dips in the absorption spectra. The amplitude of the reduced-absorption dips increases with increasing the power density, while the amplitude of the absorption profile as a whole decreases due to the saturation of the transition.

It is worthy to mention another interesting property of atomic absorption resulting from Dicke effect observed in the optical domain of the spectrum. The first spectrum shown in Fig. 6 represents the absorption of Cs layer with thickness $L=\lambda / 2$, and the second for $L=\lambda$. Interesting peculiarity is that the twofold enhanced thickness of Cs layer does not result in the absorption doubling at the centers of the optical transitions. Even in opposite - at the center of the strongest 4-5 transition, the absorption at $L=\lambda / 2$ is larger than that $L=\lambda$, under the condition of atomic transition saturation. The reason for that is the coherent response of the medium as a result of the Dicke effect, which is the strongest at $L=\lambda / 2$ and the hyperfine transition saturation at $\mathrm{L}=\lambda$.

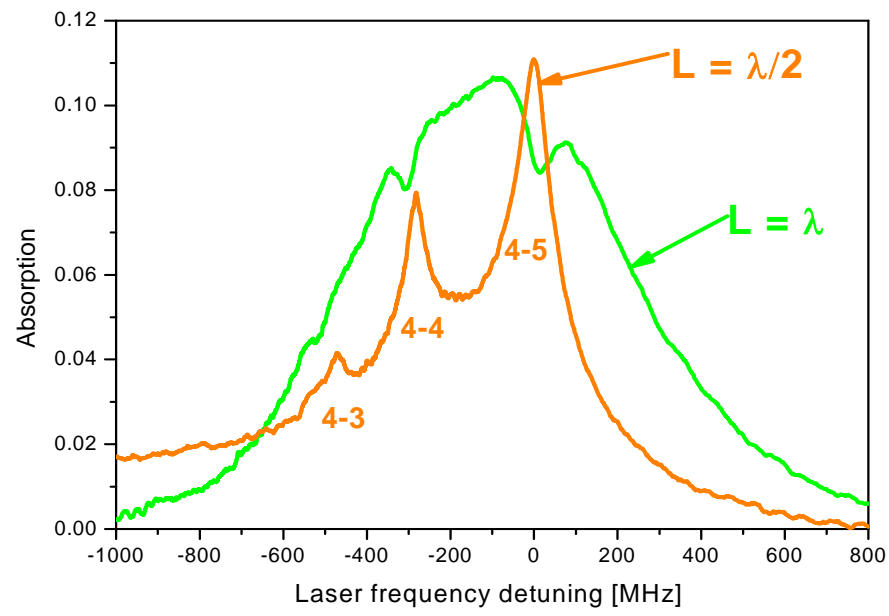

Fig. 6. Absorption spectra of two nanometric vapor layers with thicknesses $L=\lambda / 2$ and $\mathrm{L}=\lambda$. Nanometric change in the ETC thickness results in a principle modification of the absorption spectrum. 
For both ETC thicknesses $(L=\lambda / 2$ and $L=\lambda$ ), a detailed experimental study is performed [Varzhapetyan], related to the saturation with light intensity of all hyperfine transitions of the $\mathrm{D}_{2}$ line of Cs. Sub-Doppler features centered at the resonance frequency of the hyperfine transitions have been observed for all used light intensities. Substantial changes in the amplitude and width of the sub-Doppler resonance for individual hyperfine transitions occur as a function of the intensity of the incident laser radiation. The absorption spectra of the transitions $6 \mathrm{~S}_{1 / 2}\left(\mathrm{~F}_{\mathrm{g}}=3\right) \rightarrow 6 \mathrm{P}_{3 / 2}\left(\mathrm{~F}_{\mathrm{e}}=2,3,4\right)$ are presented in Fig.7a,b, and of the transitions $6 \mathrm{~S}_{1 / 2}\left(\mathrm{~F}_{\mathrm{g}}=4\right) \rightarrow 6 \mathrm{P}_{3 / 2}\left(\mathrm{~F}_{\mathrm{e}}=3,4,5\right)$ - in Fig.8a,b.

For each value of light intensity, one could immediately note the significant difference observed between the absorption spectra at $L=\lambda / 2$ (a) and these at $L=\lambda(b)$. Confirming the results of Ref. [Dutier, 2003, a; Sarkisyan, 2004], at $L=\lambda / 2$ strong narrowing of the hyperfine transition absorption is obtained. All hyperfine transitions are well resolved. Fig.7a and Fig.8a show significant power broadening of the observed sub-Doppler structures.

(a)

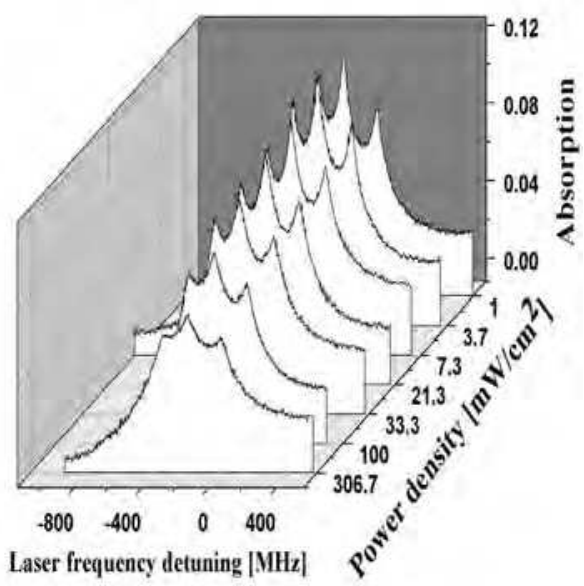

(b)

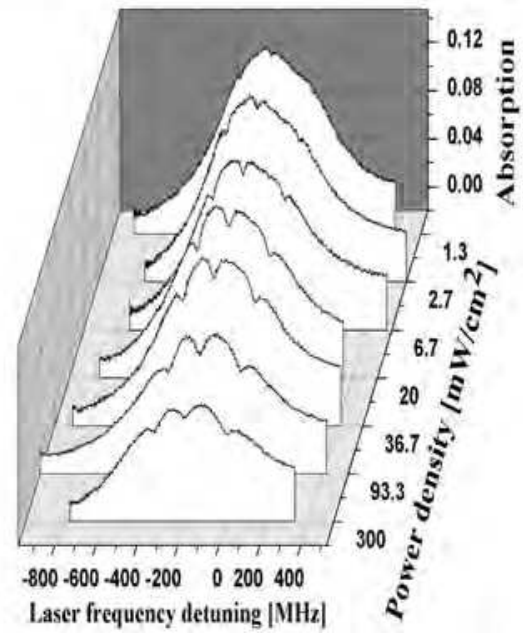

Fig. 7. Absorption spectra of the $\mathrm{F}_{\mathrm{g}}=3$ set of hyperfine transitions obtained for different laser intensities at $L=\lambda / 2$ (a) and $L=\lambda(b)$. The atomic source temperature is $T=100^{\circ} \mathrm{C}$. ECDL is used with spectral width of $3 \mathrm{MHz}$.

For $\mathrm{L}=\lambda$, the coherent Dicke narrowing vanishes and as a result of the Doppler broadening the hyperfine structure is not resolved, which is evident at the lowest light intensities. At ETC thickness equal to $\lambda$, dips of reduction of the absorption appear. The origin of these dips is related to the fact that processes like saturation of the atomic transition and optical pumping to the ground level non-interacting with the laser light can be completed only for atoms with large enough time of interaction with the laser light, giving rise to highly velocity-selective dips in the absorption spectra (Fig.7b and Fig.8b). These sub-Doppler resonances of reduced absorption suffer strong power broadening. For the hyperfine transitions starting from $F_{g}=3$ level and for laser power density of $300 \mathrm{~mW} / \mathrm{cm}^{2}$ the width of the reduced absorption resonances is about $60 \mathrm{MHz}$. 
Consequently, by utilizing ETC and low-intensity irradiation, it is possible to distinguish two very important cases: (i) cell thickness $L=\lambda / 2$ and (ii) $L=\lambda$. In the first case, the coherent response of the atomic dipoles to the light results in maximal narrowing of the hyperfine transitions profile, while in the second, the contribution of the slow atoms is concealed by destructive interference, leaving a Doppler-broadened absorption spectrum only.

(a)

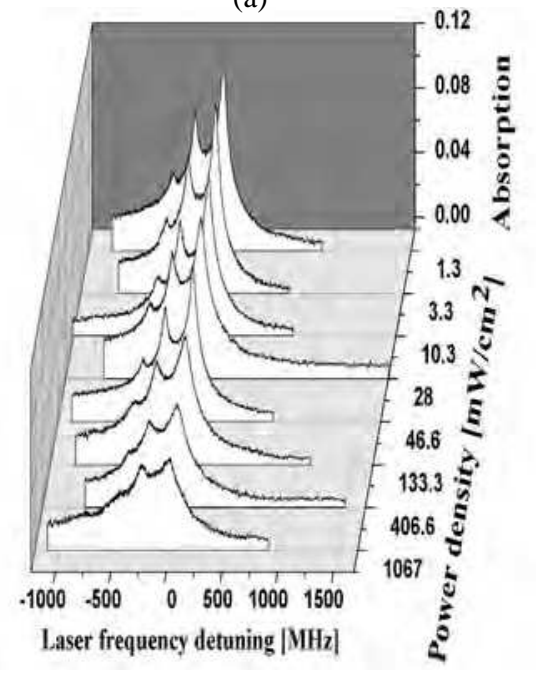

(b)

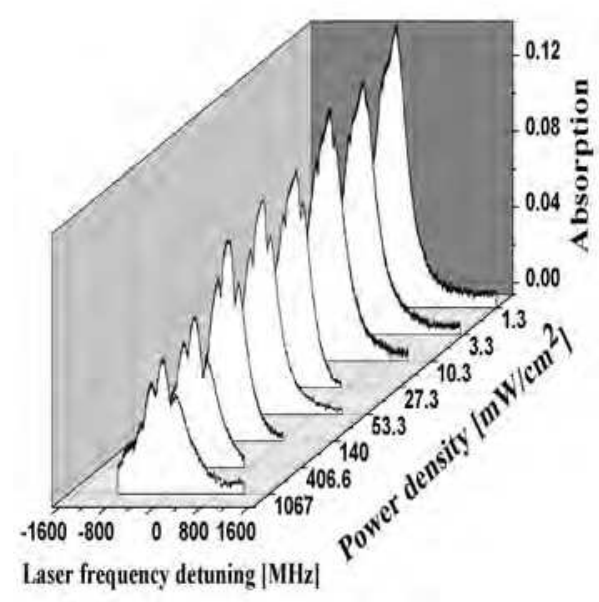

Fig. 8. Absorption spectra of the $\mathrm{F}_{\mathrm{g}}=4$ set of hyperfine transitions obtained for different laser intensities at $L=\lambda / 2$ (a) and $L=\lambda(b)$. The atomic source temperature is $T=119{ }^{\circ} \mathrm{C}$. ECDL is used with spectral width of $3 \mathrm{MHz}$.

\subsubsection{Absorption spectra of atoms confined in ETC with $L=m \lambda$ $(\mathrm{m}=0.5,1,1.5,2,2.5,3)$}

In this section, the discussion is expanded to ETCs with larger thickness - up to several microns. As discussed above the Dicke narrowing vanishes for $L=\lambda$. However further enlargement of $\mathrm{L}$ can result in the Dicke narrowing revival. Collapse and revival of the coherent Dicke narrowing of atomic transition absorption profiles were demonstrated in Ref. [Dutier, 2003, a; Sarkisyan, 2004], revealing the transition width quasi periodicity as a function of optical cell thickness $L$, with minima at $L=(2 n+1) \lambda / 2$ (n - integer; $\lambda$ - light wavelength).

The Cesium-vapor-layer transmission spectrum is measured for different cell thicknesses and low and high intensities of the light [Cartaleva, 2009]. In Fig.9, the transmission spectrum of the $6 \mathrm{~S}_{1 / 2}\left(\mathrm{~F}_{\mathrm{g}}=4\right) \rightarrow 6 \mathrm{P}_{3 / 2}\left(\mathrm{~F}_{\mathrm{e}}=3,4,5\right)$ set of hyperfine transitions is presented, for the ETC thickness $\mathrm{L}=\mathrm{m} \lambda(\mathrm{m}=0.5,1,1.5,2,2.5,3)$.

For low light intensity $\left(0.2 \mathrm{~mW} / \mathrm{cm}^{2}\right.$, where the saturation of optical transitions can be neglected) and $\mathrm{m}=0.5$, one can see (Fig.9a) that all hyperfine transitions are well resolved and the Doppler broadening of the transmission (absorption) profiles is very small. Keep in mind that the Doppler width of the hyperfine transitions of Cs in conventional cells at room temperature is about $400 \mathrm{MHz}$. In the $\mathrm{L}=0.5 \lambda$ case, the absorption at the resonance 
frequency is strongly enhanced due to the existence of two similar in their result but different in their nature processes. As above discussed, the first process is connected to the anisotropy of atom-light interaction. As in our experiment the laser beam diameter (about 1 $\mathrm{mm}$ ) largely exceeds the cell thickness, the time interaction between an atom and the laser radiation is different depending on the atomic velocity direction. For the ETC, it is assumed that atoms lose their excitation when hitting the cell wall. The second process arises as a consequence of the coherent nature of the atomic ensemble emission, which leads to relative enhancement of absorption at the optical transition center compared to its wings.
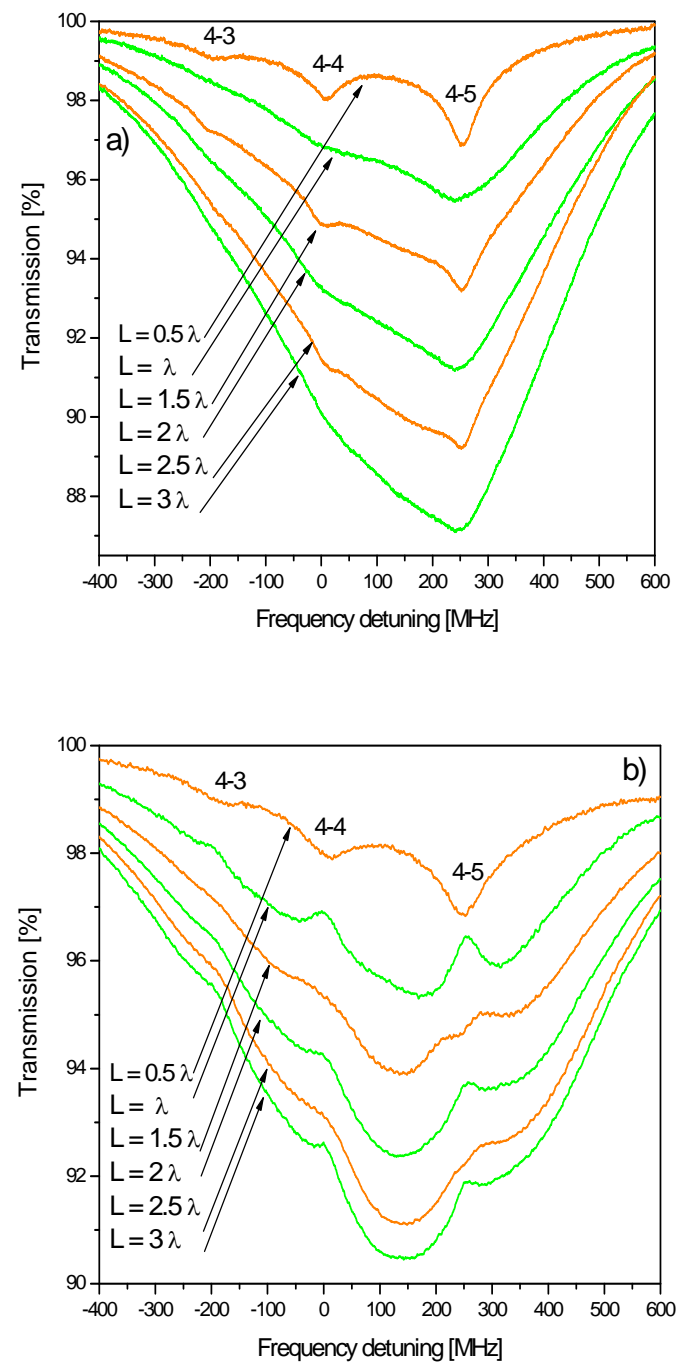

Fig. 9. Transmission spectra of the $F_{g}=4$ set of transitions, for low $\left(\mathrm{a}, 0.2 \mathrm{~mW} / \mathrm{cm}^{2}\right)$ and high (b, $20 \mathrm{~mW} / \mathrm{cm}^{2}$ ) light intensities. Monomode laser used is with spectral width of $15 \mathrm{MHz}$. 
The quasi periodic nature of the second process - periodical revival of Dicke narrowing (its relative contrast decreases with respect to the broad pedestal, which increases proportionally to L) with the ETC thickness enlargement - is well recognizable in Fig.9a (for $\mathrm{m}=0.5,1.5,2.5$; orange traces). While the Dicke coherent narrowing has it maximum at $\mathrm{m}=0.5$, for the first Dicke revival case $(\mathrm{m}=1.5)$, the well pronounced narrow, enhanced absorption peaks are superimposed on a large pedestal. Simple calculation shows that the revival of narrowing should be observable up to thickness $L_{\max }=u t$, where $\mathrm{u}$ is the mean thermal velocity of Cs atoms $(\sim 250 \mathrm{~m} / \mathrm{s})$, and $t$ is the excited state lifetime ( $30 \mathrm{~ns})$, that is $L_{\max } \sim 7.5 \mu \mathrm{m}$ [Briaudeau, 1998].

At $\mathrm{m}=1,2,3$ (green traces) however, the coherent Dicke narrowing vanishes, and because of that these ETC thicknesses are determined as positions of Dicke effect collapsing. Hence, due to the Doppler broadening the optical transitions are not resolved even at low light intensity [Briaudeau, 1996].

Increasing the irradiation power (Fig. 9b, $\mathrm{m}=0.5$ ), some broadening of the sub-Doppler absorption profiles is observed due to saturation. At $m=1,2,3$ (green traces), well pronounced narrow peaks in transmission (reduced absorption dips) are observed at the hyperfine transition centers. The amplitude and contrast of the narrow transmission peaks reduce with the ETC thickness enlargement. It has been shown that the transmission peak amplitude increases with increasing laser power density [Andreeva, 2007, b; Sarkisyan, 2004].

For $\mathrm{m}=1.5,2.5$ and for the completely closed $\mathrm{F}_{\mathrm{g}}=4 \rightarrow \mathrm{F}_{\mathrm{e}}=5$ transition, a small absorption peak (i.e. dip in the transmission) appears superimposed on the velocity selective absorption dip. Such a narrow absorption peak (Dicke signal) does not appear for the open transitions under the same experimental conditions. This difference can be related to the fact that for the open transitions part of the atoms is transferred to the $F_{g}=3$ level and this part consists mainly of slow atoms. Thus, slow atoms are lost for the absorption and they can not participate in the formation of the coherent signal. One can conclude that for open transitions, mainly fast atoms contribute to the Dicke effect. In opposite, for the completely closed transition the slow atoms give enhanced contribution to Dicke signal, resulting in increased absorption in a narrow spectral region, centered at the optical transition. In support of this statement one can notice that due to the contribution of Dicke effect to the $\mathrm{F}_{\mathrm{g}}=4 \rightarrow \mathrm{F}_{\mathrm{e}}=4$ transition absorption (Fig. $9 \mathrm{~b}, \mathrm{~m}=1.5$ ), the amplitude of the velocity selective absorption dip is sufficiently less and its width is larger than that for the same transition but at $\mathrm{m}=1,2$. Nevertheless, this contribution is not enough for narrow absorption peak formation, as it happens for the $\mathrm{F}_{\mathrm{g}}=4 \rightarrow \mathrm{F}_{\mathrm{e}}=5$ transition (Fig.9b, $\mathrm{m}=1.5$ ) under the same experimental conditions.

To analyze in more precise way the Dicke effect for open transitions, the transmission spectra are presented of the $6 \mathrm{~S}_{1 / 2}\left(\mathrm{~F}_{\mathrm{g}}=3\right) \rightarrow 6 \mathrm{P}_{3 / 2}\left(\mathrm{~F}_{\mathrm{e}}=2,3,4\right)$ set of transitions (Fig.10), which contains only open transitions, i.e. atomic transitions suffering population loss to level noninteracting with the laser field. The population loss is caused by hyperfine and/or Zeeman optical pumping processes, based on the spontaneous decay from the excited level. Moreover, a very recently developed laser system is used with DFB laser source and extremely low noise current controller. This system allowed us to achieve strong improvement of the spectral resolution and signal-to-noise ratio. 
From Fig.10 it can be seen that narrow and well pronounced enhanced transmission peaks can be observed at much lower than in Fig.9b light intensity. This makes possible simultaneous observation of Dicke enhanced absorption narrow features at $\mathrm{m}=1.5$ (first Dicke revival) and enhanced transmission peaks for $m=1,2,2.5,3$. Note also the Dicke effect contribution to some broadening and amplitude reduction of resonances at $\mathrm{m}=2.5$.

Consequently, based on the transmission spectrum of ETC with thickness of few microns, a frequency reference can be developed with sub-Doppler-width precision. It should be stressed that the transmission spectrum is measured by extremely simple and robust, single beam optical system. As the narrow resonances are centered at the hyperfine transitions, the potential accuracy of the frequency reference is high.

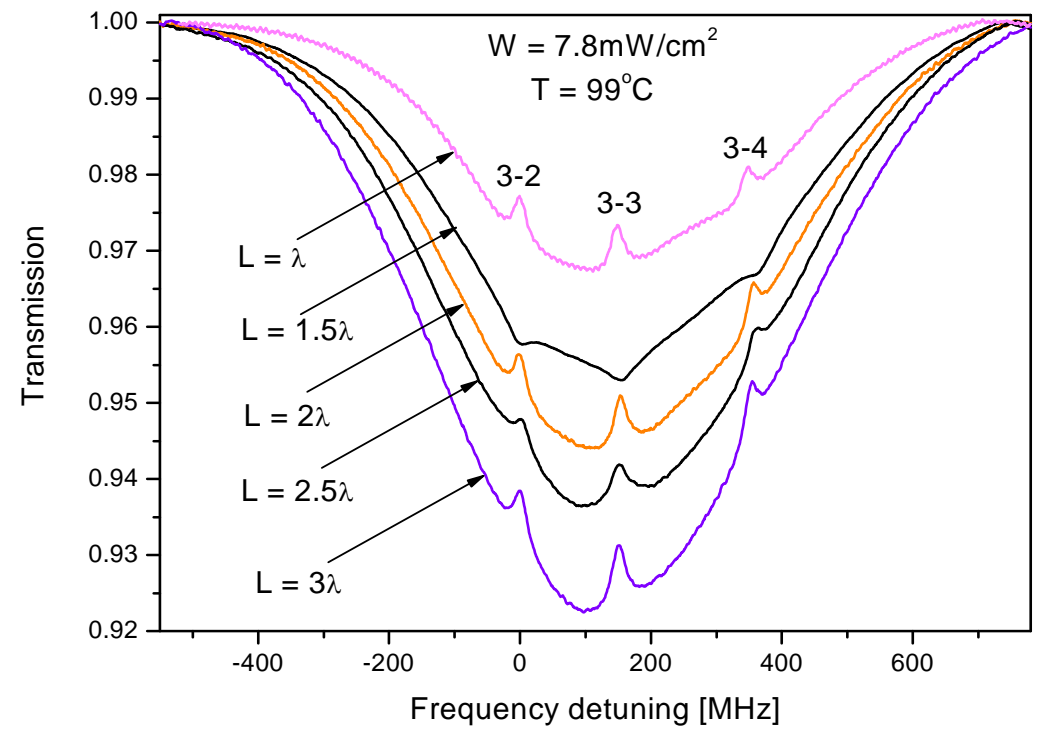

Fig. 10. Transmission spectra of the $F_{g}=3$ set of transitions, for five different ETC thicknesses and $\mathrm{W}=7.8 \mathrm{~mW} / \mathrm{cm}^{2}$. The low intensity noise DFB laser system is used with spectral width of $2 \mathrm{MHz}$.

As the contrast of the enhanced transmission peaks (Fig.10) is largest at $\mathrm{L}=\lambda$, a measurement is performed of the broadening by the light intensity of the enhanced transmission peaks observed in the transmitted spectrum, for three light intensities (Fig.11). It can be seen that the resonance broadening with light intensity is not fast, and very good signal to noise ratio can be provided at resonance width of about $10 \mathrm{MHz}$. Note that the measured width is about 40 times less than the Doppler width of the hyperfine transition and close to the natural width of the transition $(6 \mathrm{MHz})$. It is worthy to point out that the lifetime of the excited state for atoms flying close to parallel to laser beam is significantly reduced due to the small ETC thickness, determining about $50 \mathrm{MHz}$ homogeneous width of atomic transition. However, based on "slow" atoms contribution much more narrow experimental structure is observed. 
Recently developed laser system with low intensity noise in the light emission and narrow spectral width allowed us to study in more detail the difference between the saturation of open and closed transitions under conditions of very well expressed Dicke revival, namely at $\mathrm{L}=1.5 \lambda$. The experimentally obtained spectra are shown in Fig.12, for larger interval of used light intensities than that presented in Fig.9. For an intensity of $1.8 \mathrm{~mW} / \mathrm{cm}^{2}$, one can distinguish all three hyperfine transitions due to the small Dicke dip in transmission, centered at each atomic transition (Fig.12). With the light intensity enhancement, a reduction of the amplitude of the narrow Dicke features occurs for both open $F_{g}=4 \rightarrow F_{e}=3,4$ transitions. Further on saturation dips around the atomic transition centers start to appear. These dips grow in amplitude and their width with the light intensity.

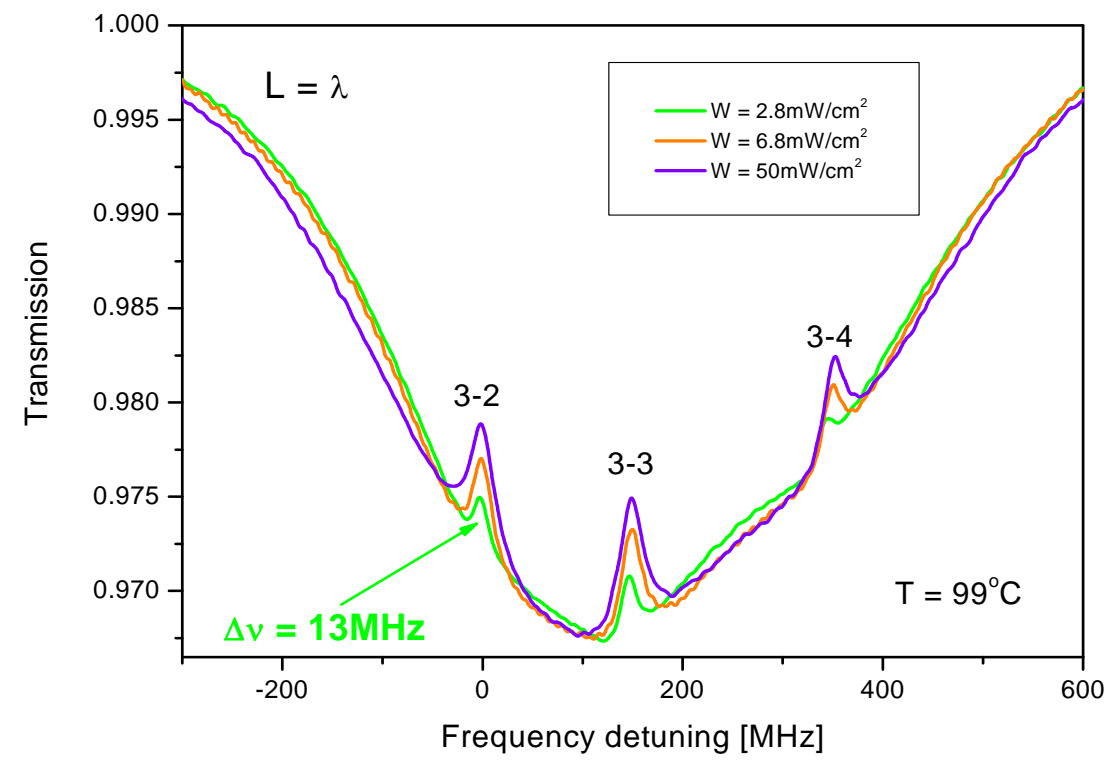

Fig. 11. Transmission spectra at three light intensities, for the $F_{g}=3$ set of transitions and $\mathrm{L}=\lambda$. DFB laser system is used with spectral width of $2 \mathrm{MHz}$.

However, for the closed $\mathrm{F}_{\mathrm{g}}=4 \rightarrow \mathrm{F}_{\mathrm{e}}=5$ transition, the saturation dip is not the only subDoppler feature that is recognizable in the absorption spectrum. In the position of the transition center, one can see remaining even at highest used intensity the Dicke absorption peak superimposed on the saturation dip. This peak is observable at all intensities, within the intensity range explored in the experiment. As above discussed, the narrow absorption peak originates from the coherent atom light interaction, and it is associated with the Dicke narrowing. Increasing the light intensity, it can be seen that the Dicke signal does not change notably its amplitude even it falls in the saturation dip of the transition, which grows in amplitude. Note also that the width of Dicke enhanced absorption resonance does not change significantly with light intensity.

When comparing the open and closed (in terms of optical pumping) transitions, one can see that the saturation peak of enhanced transmission appears simultaneously for both types of 


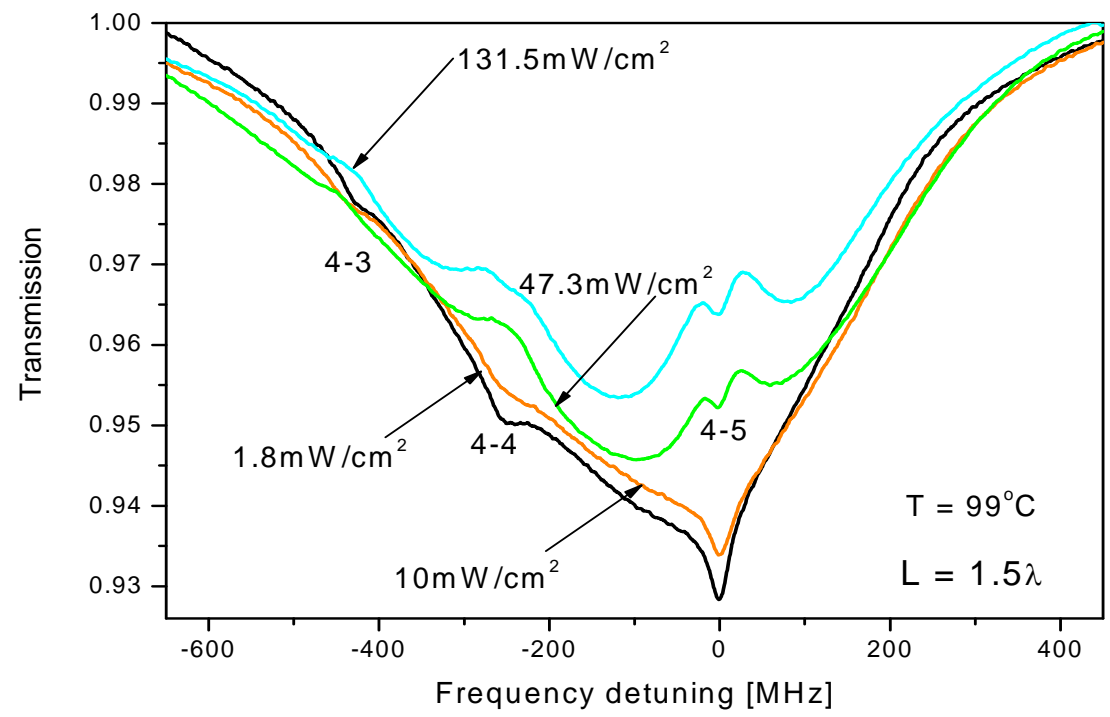

Fig. 12. Transmission spectra of the $\mathrm{F}_{\mathrm{g}}=4$ set of transitions, different saturation behavior of open and closed atomic transitions under condition of Dicke effect revival. DFB laser system is used with spectral width of $2 \mathrm{MHz}$.

transitions. The behavior of the peak amplitude and width dependences on the light intensity is also similar. However, for higher light intensity, the narrow Dicke signal is not pronounced for the open transitions, while the narrow Dicke dip in transmission is always present, in the closed transition case. The reason for such difference could be found in the transit time effects of atom light interaction, intrinsic to the nature of the ETC, combined with the optical pumping (in three-level system) or with the saturation effects in two-level system. It is well known [Dutier, 2003, a; Sarkisyan, 2004; Briaudeau, 1998] that the Dicke narrowing results in the appearance of a narrow Dicke signal over Doppler broaden pedestal, where Dicke signal originates from the transit times effects. The significant contribution to the narrow Dicke signal comes from the slow atoms. However, namely the slow atoms suffer the highest loss due to the optical pumping to the ground-state level noninteracting with the light, which occurs in case of the open transitions. Hence at the open transitions, the Dicke signal is missing, for higher light intensities.

\subsection{Sub-Doppler resonances in the fluorescence - Experimental results}

\subsubsection{Monotonic broadening of sub-Doppler fluorescence profile with ETC thickness enlargement}

The investigation of the absorption and fluorescence of such confined media has started in the weak-intensity regime [Dutier, 2003,b], and the work was concentrated mainly on the investigation of the absorption spectra, considering that the fluorescence profiles exhibit only monotonic broadening with the cell thickness [Sarkisyan, 2004]. 


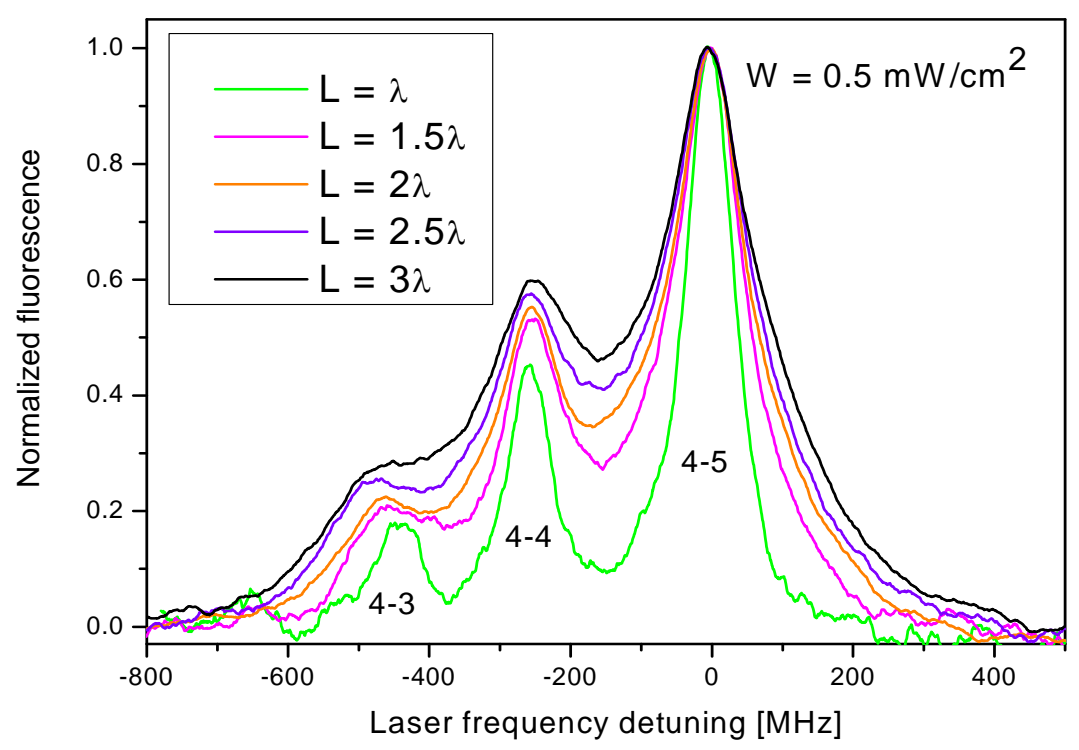

Fig. 13. Normalized fluorescence spectra of the $F_{g}=4$ set of transitions, at five different ETC thicknesses. The fluorescence profile broadening with $\mathrm{L}$ is clearly seen.

For low light intensity, our investigations confirm the monotonic broadening of the fluorescence profiles when increasing the ETC thickness. In Fig.13, the normalized fluorescence spectra of the $6 \mathrm{~S}_{1 / 2}\left(\mathrm{~F}_{\mathrm{g}}=4\right) \rightarrow 6 \mathrm{P}_{3 / 2}\left(\mathrm{~F}_{\mathrm{e}}=3,4,5\right)$ transitions are presented, for low irradiating power and $\mathrm{L}=\mathrm{m} \lambda$ with $\mathrm{m}=1,1.5,2,2.5,3$. Due to the small ETC thickness (for $\mathrm{m}=1$ ), atoms with velocity normal to the cell windows give very small contribution to the fluorescence signal compared to atoms flying parallel to the ETC windows, which have much larger time of interaction with the laser beam. Confirming previous investigations [Sarkisyan, 2001; Andreeva, 2007, b; Sarkisyan, 2004] we present here very well resolved fluorescence profiles of the three hyperfine transitions, for $L=\lambda$. The reason is that the fluorescence signal comes mainly from "slow" atoms, whose velocity projection on the light beam is small enough to allow time for absorption of a photon and subsequent spontaneous emission before collisions with the cell wall. With the ETC thickness enhancement broader velocity class atoms have enough time to complete the fluorescence emission, which leads to monotonic rising of the fluorescence profile width. In this way, the resolution of the hyperfine transitions at $L=3 \lambda$ is much worse than that for $L=\lambda$.

\subsubsection{Appearance of a narrow structure in the sub-Doppler fluorescence profiles: Different behavior of open and closed transitions}

Further study of the ETC fluorescence spectra at $L=\lambda$ has demonstrated [Andreeva, 2007, b] that tiny saturation dip of reduced fluorescence appears in the narrow fluorescence profiles when irradiating the ETC with higher-intensity laser light. These saturation dips appear for all open, in terms of hyperfine and Zeeman optical pumping, hyperfine transitions of $\mathrm{Cs}_{2} \mathrm{D}_{2}$ line. No saturation dip was reported there in the fluorescence of the $F_{g}=4 \rightarrow F_{e}=5$ 
transition, which is the only completely closed transition on the $\mathrm{D}_{2}$ line of Cs. As for ETC with $L=\lambda$ the fluorescence dips are of extremely small amplitude (slightly higher than the experimental noise), in Ref. [Andreeva, 2007, b; Varzhapetyan] their examination has been performed by phase sensitive registration. Later on, it has been shown that with the enhancement of ETC thickness the reduced fluorescence dips increase their amplitudes [Cartaleva, 2009]. However, there the spectral width of the emission of the used diode laser system was about $15 \mathrm{MHz}$, which was a reason for significant broadening of the dips observed in the fluorescence and strong reduction of their amplitude. Due to this, we present here our recent experimental results, obtained by means of the narrow band and low noise laser system. The significant difference between the behavior of the open and closed transitions is illustrated in Fig.14, for ETC with thickness $L=1.5 \lambda$. It can be seen that at both $\mathrm{F}_{\mathrm{g}}=4 \rightarrow \mathrm{F}_{\mathrm{e}}=3,4$ open hyperfine transitions good-amplitude and narrow reduced fluorescence dips occur.

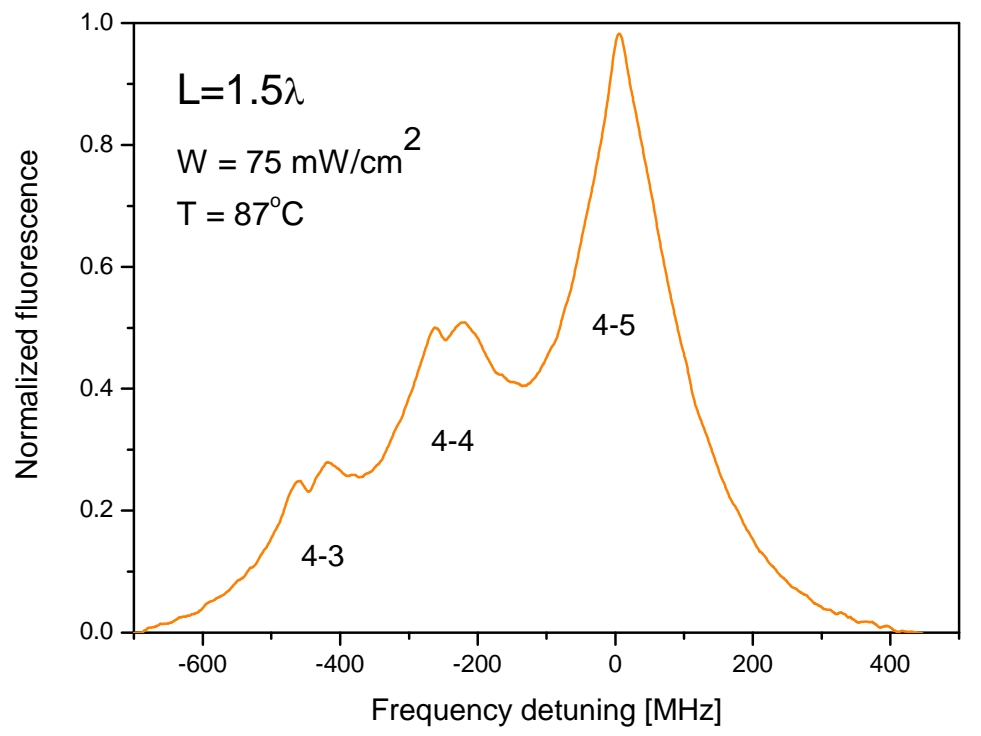

Fig. 14. Fluorescence spectrum, observed in ETC with thicknesses $L=1.5 \lambda$, at the $F_{g}=4$ set of transitions. The 4-5 transition is the closed one, while the 4-4 and 4-3 transitions are open transitions.

Similar to the absorption at lower light intensity, the fluorescence on the open transitions suffers loss from the velocity selective optical pumping, which results in narrow reducedfluorescence dips appearing at the centre of the transitions. Thus, the ETC fluorescence profiles, which are intrinsically narrower than the absorption profiles, exhibit velocity selective dips for higher light intensity values.

The situation is different for the completely closed $F_{g}=4 \rightarrow F_{e}=5$ transition, namely no dip occurs at the atomic transition centre (Fig.14). In opposite, the top of the highest probability 
optical transition is very sharp, and one can assume that the fluorescence profile of the closed transition involves a small narrow peak at its centre.

The processes leading to the dip and the peak formations can be illustrated as follows.

For the open transitions (see as an example the $F_{g}=3 \rightarrow F_{e}=2$ transition, Fig.15a), both two-level-system saturation and three-level-system optical pumping deplete the atomic population of the coupled by the light ground Zeeman sublevels. The result of this velocity selective depleting is the narrow, reduced fluorescence dip centered at the open transition.

The situation is different for the completely closed $F_{g}=4 \rightarrow F_{e}=5$ transition (Fig.15b). Here, the two-level-system saturation process decreases the ground Zeeman sublevel populations, while the fluorescence increases the sublevel population in a narrow frequency interval. Moreover, the fluorescence is enhanced by the fact that the light accumulates atomic population on Zeeman sublevels with the largest absorption probability [Andreeva, 2002] i.e. a large portion of the redistributed by the light atoms stays in the most absorbing magnetic sub-level of $F_{g}=4$ level. Thus, the summary contributions of both processes result in a small narrow peak formation.


Fig. 15. Illustration of saturation and optical pumping for the open in terms of Zeeman optical pumping $\mathrm{F}_{\mathrm{g}}=3 \rightarrow \mathrm{F}_{\mathrm{e}}=2$ transition (a) and the completely closed $\mathrm{F}_{\mathrm{g}}=4 \rightarrow \mathrm{F}_{\mathrm{e}}=5$ transition (b). 


\subsubsection{The behavior of the narrow structure in the fluorescence profiles with the ETC thickness enlargement}

In Ref. [Cartaleva, 2009], the examination of the saturated regime in the fluorescence was expanded to ETC thickness up to $\mathrm{L}=3 \lambda$. It has been shown that the amplitude of the narrow dip in the fluorescence profile of the open transitions increases with the ETC thickness. Starting from $L=2 \lambda$, the fluorescence profile of the completely closed transition exhibits small plateau, centered at the optical transition.

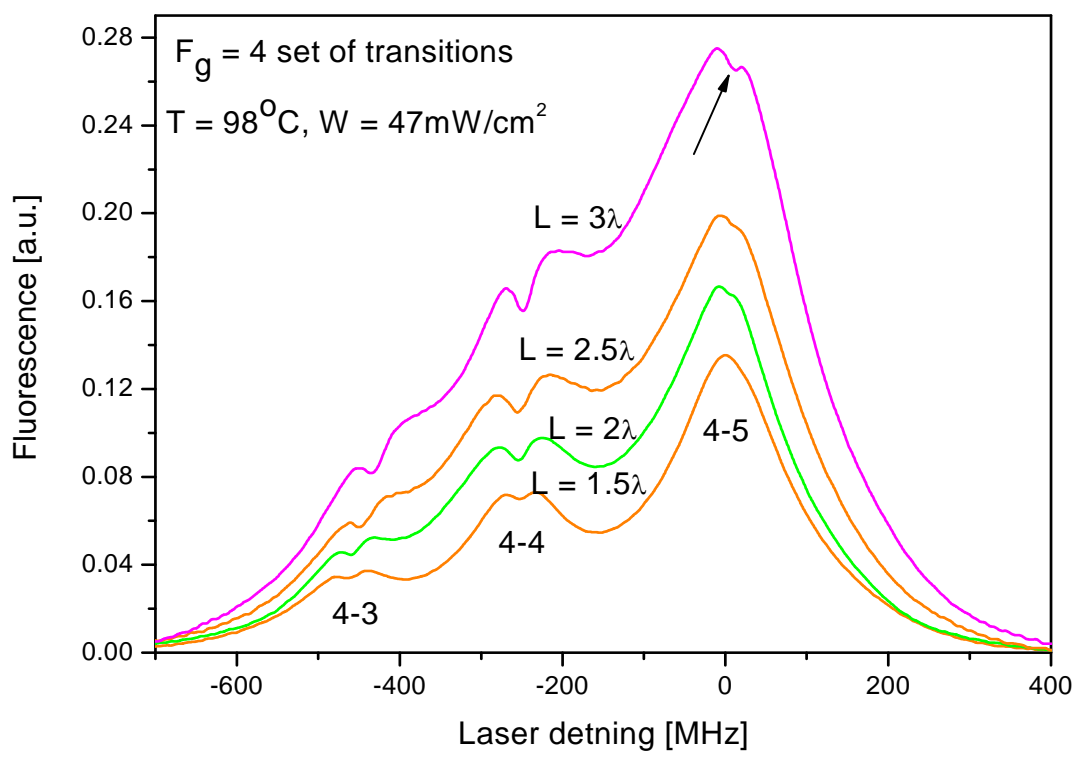

Fig. 16. Fluorescence spectra at the $\mathrm{F}_{\mathrm{g}}=4$ set of transitions, observed in ETC with thickness $\mathrm{L}=1.5 \lambda, 2 \lambda, 2.5 \lambda, 3 \lambda$. The ETC temperature $\mathrm{T}=98^{\circ} \mathrm{C}$ and the light intensity $\mathrm{W}=47 \mathrm{~mW} / \mathrm{cm}^{2}$. The new dip in the profile of the $\mathrm{F}_{\mathrm{g}}=4 \rightarrow \mathrm{F}_{\mathrm{e}}=5$ transition is marked by an arrow.

Very recently, the first observation of a narrow, reduced fluorescence feature also in the profile of the completely closed $\mathrm{F}_{\mathrm{g}}=4 \rightarrow \mathrm{F}_{\mathrm{e}}=5$ transition has been reported [Cartaleva, 2011], obtained by means of the developed narrow-band laser system. To illustrate the new structure, the ETC fluorescence spectra for the $\mathrm{F}_{\mathrm{g}}=4$ set of transitions are presented, at cell thickness $\mathrm{L}=1.5 \lambda, 2 \lambda, 2.5 \lambda, 3 \lambda$ (Fig.16). The two open $\mathrm{F}_{\mathrm{g}}=4 \rightarrow \mathrm{F}_{\mathrm{e}}=3,4$ transitions show reduced fluorescence dips at the central frequency of each fluorescence profile, for all examined ETC thicknesses. The dip amplitudes increase noticeably with the cell thickness. This result differs from that reported in Ref.[Cartaleva, 2009], where the dip formation starts from $L=2 \lambda$, for the open transitions. We attribute this difference to the fact that in the present work the spectral width of the laser line is significantly narrower $(2 \mathrm{MHz})$ than that used in [Cartaleva, 2009] (15 MHz). Thus, the observation of the narrow dip in the fluorescence depends very critically on the laser line width, which was not as critical for the transmission spectra. The amplitude of the dips increases with the cell thickness, due to the contribution of larger number of atoms to its formation. At larger cell thickness, the time of 
flight of the atoms between the cell walls increases, which broadens the atomic velocity interval of light-emitting atoms. It can be seen that the emitting atom number enlargement with L makes higher contribution to the dip amplitude than to its width.

The used in the present experiment DFB laser system allows observation of a new dip in the profile of the fluorescence of the completely closed $F_{g}=4 \rightarrow F_{e}=5$ transition (Fig.16, $L=3 \lambda$ ). With a similar set of the ETC thicknesses [Cartaleva, 2009] only a small plateau has been observed at the $\mathrm{F}_{\mathrm{g}}=4 \rightarrow \mathrm{F}_{\mathrm{e}}=5$ transition up to $\mathrm{L}=3 \lambda$. In Fig.16, similar plateau can be seen for $L=2 \lambda, 2.5 \lambda$. In Ref. [Cartaleva, 2009], the formation of the plateau for the completely closed transition instead the dip for open transitions has been explained by the saturation and optical pumping processes. For the open transitions, both processes deplete the atomic population of ground Zeeman sub-levels coupled by the light. However, in case of the $\mathrm{F}_{\mathrm{g}}=4 \rightarrow \mathrm{F}_{\mathrm{e}}=5$ transition, while the two-level-system saturation process decreases the ground Zeeman sublevel populations, the fluorescence increases the sub-level population in a narrow frequency interval. The summary contributions of both processes can result in the small plateau formation (see Fig.16, $\mathrm{L}=2 \lambda, 2.5 \lambda$ ).

Concerning the new dip formation [Cartaleva, 2011], the assumed physical processes behind this are based on the degeneracy of the two-level system. As has been shown in Ref.[Andreeva, 2002], if the $F_{g}=4 \rightarrow F_{e}=5$ transition is excited by linearly polarized light (as in our experiment), $C$ s atoms accumulate on the $F_{g}=4$ Zeeman sublevels with the highest probability of excitation, i.e. the highest fluorescence is expected for slow atoms [Cartaleva, 2009]. However, in the case of depolarization of the excited level, a significant portion of slow atoms will be accumulated on the $F_{g}=4$ Zeeman sublevels with the lowest probability of excitation [Andreeva, 2002]. Hence if the two-level system is a degenerate one, the excited state depolarization will transform the completely closed system to one with effective loss in the excitation process. The reducing of the optical transition excitation rate will be the most significant for slow atoms, resulting in narrow dip formation in the fluorescence profile. The assumed depolarization of the excited level can be caused by the collisions between Cs atoms. Another reason for the excited state depolarization could be the influence of the cell window, as has been suggested in [Andreeva, 2007, a].

\subsubsection{Frequency reference based on the ETC fluorescence spectrum}

While the study of the difference between the open and closed transitions is important from basic point of view, the set of the $6 \mathrm{~S}_{1 / 2}\left(\mathrm{~F}_{\mathrm{g}}=3\right) \rightarrow 6 \mathrm{P}_{3 / 2}\left(\mathrm{~F}_{\mathrm{e}}=2,3,4\right)$ transitions is very interesting for the development of practical frequency reference. This group consists of open transitions only. Hence, for each hyperfine transition, well pronounced reduced fluorescence dip can be formed, which is seen from Fig.17. While at $L=\lambda$ the amplitude of dip is very small, it grows up significantly at $L=3 \lambda$. As the dips are observed at the centres of the optical hyperfine transitions, they can be used as frequency reference in the field of laser spectroscopy and for development of highly stabilized laser systems.

Our theoretical and experimental investigations [Vaseva] have shown that further enlargement of the ETC thickness up to $L=6 \lambda$ results in even better parameters (contrast and spectral width) of the narrow dip centered in the fluorescence profile. The enhancement of the ETC thickness up to $L=6 \lambda$ not only provides the possibility for strong reduction of the used light intensity but also allows working at much lower atomic source temperature, which is of significant importance for operating of practical frequency reference devices. In 
Fig.18, the ETC transmission and fluorescence spectra are presented for reduced atomic source temperature down to $49^{\circ} \mathrm{C}$. An additional advantage of the ETC with $\mathrm{L}=6 \lambda$ is that it exhibits even lower width of the dips observed in the fluorescence spectrum than that of the peaks in the transmission spectrum (Fig.18).

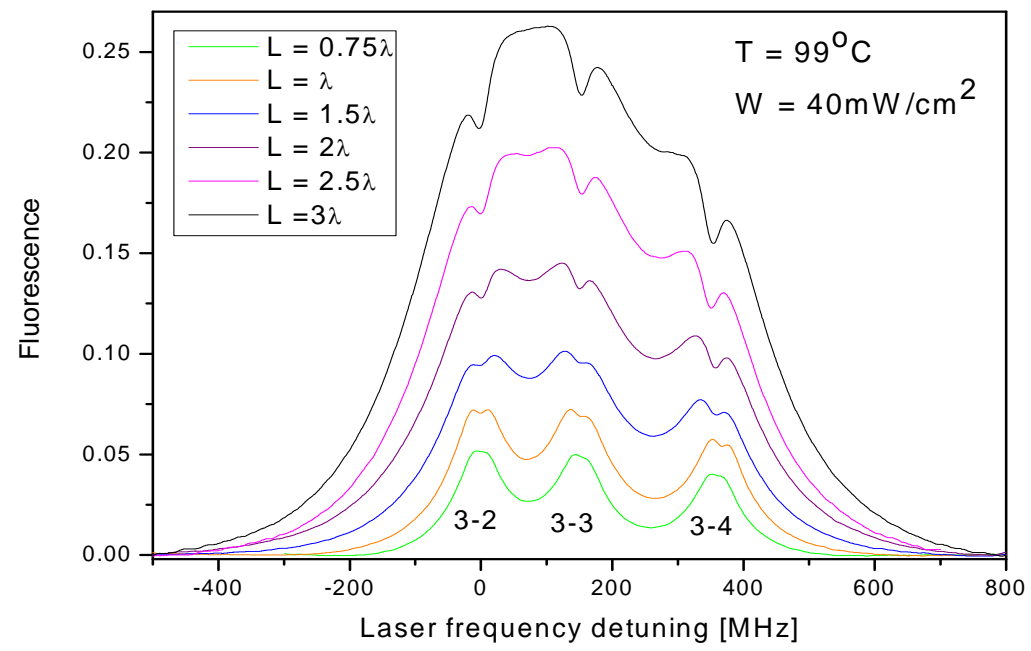

Fig. 17. Fluorescence spectra at the $\mathrm{F}_{\mathrm{g}}=3$ set of transitions, observed in ETC with thickness $\mathrm{L}=0.75 \lambda, \lambda, 1.5 \lambda, 2 \lambda, 2.5 \lambda, 3 \lambda$. The ETC temperature $\mathrm{T}=99^{\circ} \mathrm{C}$ and the light intensity $\mathrm{W}=40 \mathrm{~mW} / \mathrm{cm}^{2}$.

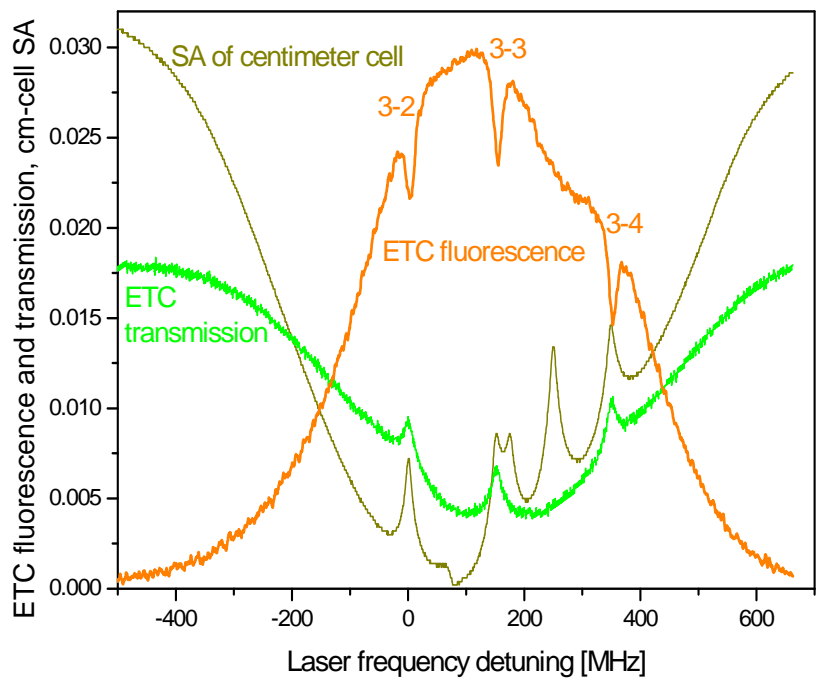

Fig. 18. Experimental fluorescence and transmission spectra $\left(\mathrm{W}=20 \mathrm{~mW} / \mathrm{cm}^{2}\right)$, for ETC with $\mathrm{L}=6 \lambda$. Cs source temperature $\mathrm{T}=49^{\circ} \mathrm{C}$. SA: Saturated absorption spectrum for conventional cell with $\mathrm{L}=2.5 \mathrm{~cm}$, at room temperature. 
For comparison with the ETC spectra, in Fig.18 a saturated absorption spectrum is shown, measured by the conventional two-counter-propagating-beam technique (see Fig.4). It is clearly seen that the saturated absorption spectrum observed in the conventional cell is more complicated than the three-narrow-resonance spectra in the ETC transmission and fluorescence. Note that even at enhanced thickness of $L=6 \lambda$, the anisotropy in the time of atom-light interaction still suppress the formation of crossover resonances, in the case of ETC. It has been shown [Sargsyan, 2008, b] that weak crossover resonance formation by single beam irradiation of micrometric cell is possible due to the reflected by the second window laser beam.

\section{Theoretical simulation of absorption (transmission) and fluorescence spectra in ETC}

The theoretical model in use is based on the Optical Bloch Equations for two-level system (closed and open), presented in Ref. [Andreeva, 2007, b]. For the open system, atomic population losses are introduced by means of a spontaneous emission to a "third level" nonexcited by the light. No atomic population losses are assumed for the closed two-level system. The atomic system consists of two levels denoted as 1 and 2, which are coupled by a laser light with frequency $\omega$ detuned by $\Delta$ from the resonant transition frequency $\omega_{21}\left(\Delta=\omega-\omega_{21}\right)$.

The control parameters are chosen to be applicable to a realistic alkali system, namely: $\gamma_{21}=5 \mathrm{MHz}, \mathrm{ku}=250 \mathrm{MHz}$, and $\mathrm{a}=1$ (closed system) or $a=0.6$ (open system). Here $\gamma_{21}$ is half the transition rate from the excited state, $\mathrm{ku}$ is the most probable Doppler shift and $\mathrm{a}$ is the probability of decay from level 2 to level 1. The Rabi frequency of the optical transition is denoted by $\Omega_{\mathrm{R}}$.

The used system of Bloch equations is the following:

$$
\begin{gathered}
v \frac{d \sigma_{21}}{d z}+D_{21} \sigma_{21}-i \frac{\Omega_{R}}{2}\left(\sigma_{11}-\sigma_{22}\right)=0 \\
v \frac{d \sigma_{22}}{d z}+\gamma_{2} \sigma_{22}-\Omega_{R} \operatorname{Im} \sigma_{21}=0 \\
v \frac{d \sigma_{11}}{d z}-\alpha \gamma_{2} \sigma_{22}+\Omega_{R} \operatorname{Im} \sigma_{21}=0
\end{gathered}
$$

where $D_{21}=\gamma_{21}+i k v-i \Delta, v$ is the atomic velocity and $\sigma_{i j}$ are the reduced density matrix elements in the rotating frame.

Atomic absorption is given by:

$$
A=\int_{0}^{\infty} G(v) \exp \left[-\left(\frac{k v}{k u}\right)^{2}\right] d v, \text { where } G(v)=\int_{0}^{L} \operatorname{Im}\left[\sigma_{21}(z, v)\right] d z
$$

The fluorescence is proportional to the quantity $\mathrm{U}$ expressed as:

$$
U=\int_{0}^{\infty} Q(v) \exp \left[-\left(\frac{k v}{k u}\right)^{2}\right] d v, \text { where } Q(v)=\int_{0}^{L}\left[\sigma_{22}(z, v)\right] d z
$$




\subsection{Simulated transmission (absorption) spectra for Cs atoms confined in ETC with $L=m \lambda(m=0.5,1,1.5,2,2.5,3)$}

Based on the briefly presented model, theoretical simulation of transmission spectra concerning open and closed atomic transitions is performed for two important cases - low and high light intensity. At low light intensity $\left(0.2 \mathrm{~mW} / \mathrm{cm}^{2}\right)$, the calculated transmission (absorption) spectra of both open (Fig.19a) and closed (Fig.19b) transitions show collapse (for $\mathrm{m}=1,2,3$ ) and revival (for $\mathrm{m}=1.5,2.5$ ) of the coherent Dicke narrowing: the narrow Dicke structure is superimposed on a broad pedestal. Note that at low light intensity, only minor difference can be found between the profiles of open and closed transitions. This can be attributed to the negligible contribution of the optical pumping effect. Comparing the experimental results presented in Fig.9a with the theoretical spectra shown in Fig.19a,b, the very good agreement can be pointed out, both for open and closed atomic transitions.

In the case of saturation regime, the behavior of the simulated closed transition differs significantly from that of the open transitions. At higher light intensity $\left(20 \mathrm{~mW} / \mathrm{cm}^{2}\right)$, in case of open transitions and $L>\lambda / 2$, our calculation shows peak in the transmission (reduced absorption) formed at the transition profile center (Fig.19c). The contrast of the theoretical absorption dips in the open transitions increases with ETC thickness, which differs from the experimental spectra (Fig.9b), where the dip contrast is lower and reduces with cell thickness. It should be pointed out that while in the theoretical simulation the laser light is considered monochromatic, in the experimental realization presented in Fig.9b the laser emission is with spectral width of $15 \mathrm{MHz}$, which is a reason for the experimental dips broadening. However, even significantly narrower, the experimental spectra at the $F_{g}=3$ set of transitions (Fig.10) also do not demonstrate strong enhancement of the absorption dip amplitude with the cell thickness.

In agreement with the experimental results (Fig.9b; $\mathrm{m}=1.5,2.5$ ), the modeling (Fig.19c; $\mathrm{m}=1.5,2.5)$ shows that in case of open transitions, the Dicke effect results only in some reduction of the absorption dip amplitude.

The closed transition behavior differs from that of the open transitions: together with the absorption dips (Fig.19d; $m=1,2,3$ ) also narrow absorption peaks superimposed on broader dips are obtained (Fig.19d; $\mathrm{m}=1.5,2.5$ ). These absorption peaks present the so called Dicke revival, which is well pronounced both in the experiment (see Fig.9b and Fig.12) and theory, in the saturated regime.

\subsection{Simulated fluorescence spectra for Cs atoms confined in ETC with $L=m \lambda$ $(\mathrm{m}=0.5,1,1.5,2,2.5,3)$}

Simulated fluorescence spectra for open and closed atomic transitions are shown in Fig.20, for six values of the ETC thickness. Comparing Fig.19 and Fig.20 one can notice that the calculated fluorescence profiles are narrower than those in the transmitted light. Fluorescence spectra exhibit narrow features, centered at the transition profiles. For the open transitions and $L>\lambda / 2$, a narrow dip in the fluorescence (Fig. 20a) appears, superimposed on the top of the sub-Doppler fluorescence profile. From the experimental fluorescence spectra presented in Fig.16 and Fig.17 one can conclude that the contrast of the experimentally observed dip in the fluorescence profile is lower than the theoretically 
obtained (Fig.20a). However, in the case of fluorescence, the contrast of both experimental and theoretical dips increases with ETC thickness.
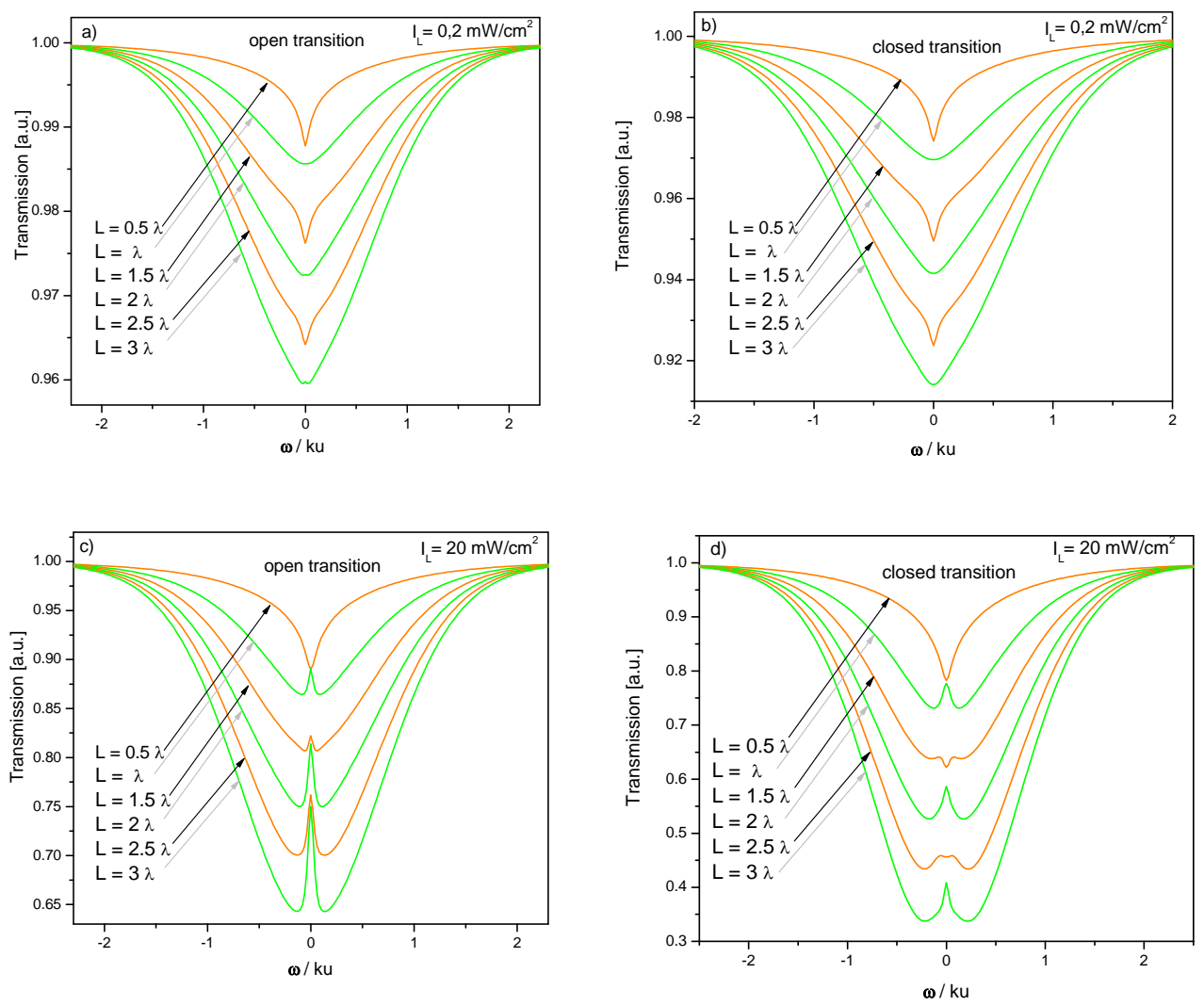

Fig. 19. Theoretical simulation of the transmission (absorption) spectra for the open $(\mathrm{a}, \mathrm{c})$ and closed $(b, d)$ transitions performed at low $(a, b)$ and high $(c, d)$ laser light intensities.

For the closed transition (Fig.20b), no dip formation can be seen in the theoretical fluorescence profile, but we observe an interesting small feature at the transition center. The fluorescence profile modification with ETC thickness starts with very small plateau on the top of the profile for $m=1$. For $m \geq 1.5$ however, a tiny narrow peak superimposed on the top of the broader fluorescence profile is clearly seen. The basic (for $L>\lambda / 2$ ) and increasing with ETC thickness difference of saturation behavior between the open and closed transitions is evident (Fig.20).

This difference can be related to the ground level population redistribution caused by the fluorescence, which is emitted by the "slow" atoms in a very narrow spectral interval. For the open transitions, this fluorescence leads to depletion of the slowest atoms from the ground Zeeman sublevels coupled to the light (see Fig.15a). The situation is opposite in case of the closed transition (see Fig.15b). Here, due to the fluorescence the slowest atoms are coming back to the coupled to the light ground Zeeman sublevels. Thus, 
for the open transition dip in the fluorescence is observed, while for the closed one a tiny peak.
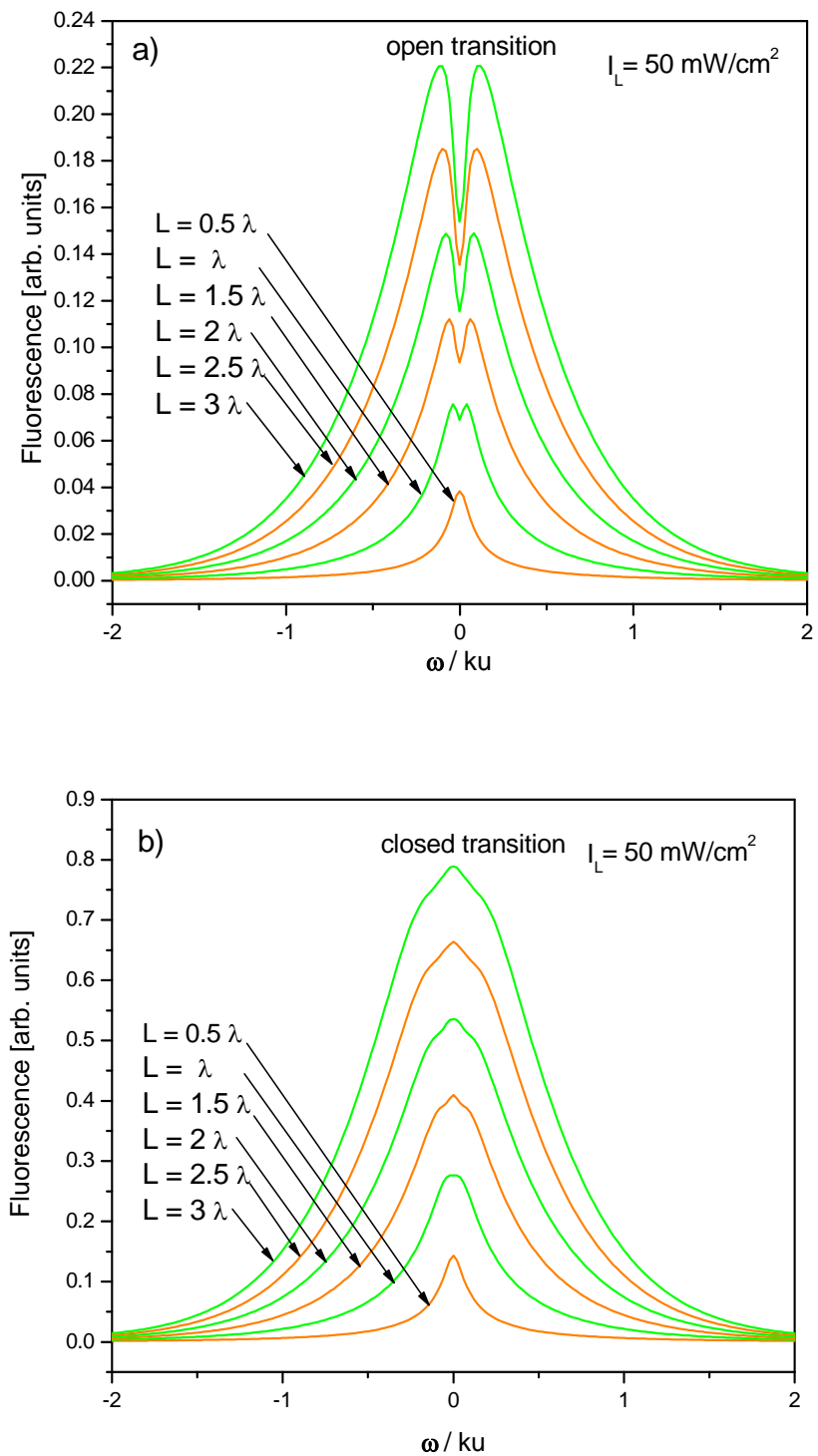

Fig. 20. Theoretical simulation of the fluorescence profile for open (a) and closed (b) transitions, at laser light intensity of $50 \mathrm{~mW} / \mathrm{cm}^{2}$.

The experimentally observed difference between open and closed hyperfine transitions is well illustrated by Fig.14. Here, the fluorescence spectrum is measured for $L=1.5 \lambda$ and for 
relatively low temperature of the atomic source $\left(87^{\circ} \mathrm{C}\right)$. Further on with the ETC thickness enlargement and Cs atom concentration enhancement (Fig.16, atomic source temperature $98^{\circ} \mathrm{C}$ and $\mathrm{L}=3 \lambda$ ), new tiny dip appears also in the fluorescence profile of the closed $\mathrm{F}_{\mathrm{g}}=4 \rightarrow \mathrm{F}_{\mathrm{e}}=5$ transition. Experimental and theoretical study is in progress, in order to clarify the physical process behind this new feature.

\subsection{Comparison of simulated absorption and fluorescence spectra for $\mathrm{Cs}$ atoms confined in ETC with $L=m \lambda(m=1,2,3,4,5,6)$}

Theoretical analysis is performed to clarify the potential of narrow dips observed in the fluorescence and absorption of open transitions for development of frequency reference. The result of the theoretical simulation is presented in Fig.21. In Fig.21a, the absorption spectra are shown, for the open transition at ETC thicknesses $\mathrm{L}=\mathrm{m} \lambda(\mathrm{m}=1,2,3,4,5,6)$ and light intensity of $20 \mathrm{~mW} / \mathrm{cm}^{2}$. Due to the population loss introduced by the hyperfine and Zeeman optical pumping, well pronounced narrow dip is observed, at the centre of the optical transition for each ETC thickness under consideration. The amplitude and the contrast of the dips are growing with the cell thickness. In case of the fluorescence (Fig.21b), one can see that the transition profiles are narrower compare to those in absorption. As discussed above, the reason is that as the laser beam diameter largely exceeds the cell thickness, the time interaction between an atom and the laser radiation is different depending on atomic velocity direction. In the narrower fluorescence profile also narrow dip occurs, exhibiting fast amplitude enhancement with the ETC thickness.

From the theoretical spectra presented in Fig.21, two important parameters were determined: the contrast and the Full Width at Half Maximum (FWHM) of the dip formed at the optical transition centre. The dependence of the dip contrast on the ETC thickness is presented in Fig.22a. It can be seen that the dip contrast observed in the fluorescence is generally lower but increases with $\mathrm{L}$ faster than that in the absorption profile. More specifically, the ratio $R_{C}$ of the dip contrast in the fluorescence $C_{F I}$ to the dip contrast in the absorption $\mathrm{C}_{\mathrm{Abs}}, \mathrm{R}_{\mathrm{C}}=\mathrm{C}_{\mathrm{Fl}} / \mathrm{C}_{\mathrm{Abs}}$, increases 2.75 times with ETC thickness enhancement from $\mathrm{L}=1000 \mathrm{~nm}$ to $\mathrm{L}=5000 \mathrm{~nm}$.

The FWHM of the dip also increases with the cell thickness (Fig.22b), but all the time the feature observed in the fluorescence is narrower than that in the absorption profile.

From the theoretical results the following conclusions can be made. A small enhancement of the ETC thickness up to $\mathrm{L}=5000 \mathrm{~nm}$ will result in about $10 \%$ rising of the contrast to FWHM ratio, in case of the fluorescence spectrum, while for the dip observed in the absorption spectrum, this ratio reduces by $30 \%$. As in general this ratio mainly determines the merit of the frequency reference, there is a strong motivation to study the fluorescence spectra for ETCs with thickness of 5-7 light wavelengths.

Consequently, both narrow dips in the absorption and fluorescence can be used for development of frequency reference. The approach based on the absorption spectrum will have better parameters at ETC with $\mathrm{L}=\lambda$, will use very low light intensity and will require atomic source heating to about $100^{\circ} \mathrm{C}$. Using the fluorescence spectrum will allow two-time reduction of the source temperature and will relax to some extent the complexity of ETC building, requiring slightly higher light intensity. 
Some possible applications such as magnetometer with submicron local spatial resolution and tunable atomic frequency references based on the ETC are described in Ref. [Sargsyan, 2008, a].
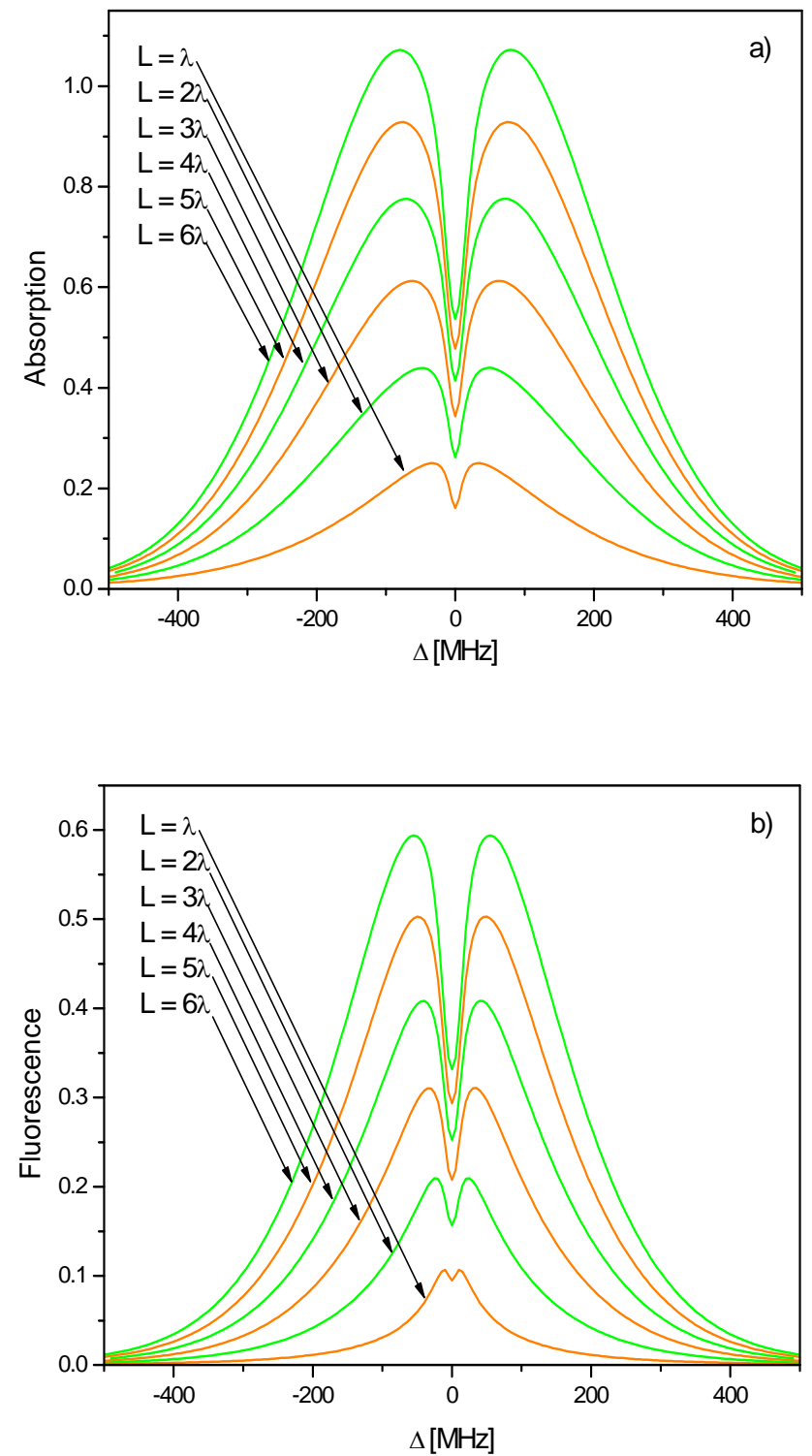

Fig. 21. Theoretical calculations of absorption (a) and fluorescence (b) spectra for open transition, and for $\mathrm{L}=\mathrm{m} \lambda$, with $\mathrm{m}=1,2,3,4,5,6$. The light intensity is of $20 \mathrm{~mW} / \mathrm{cm}^{2}$. 

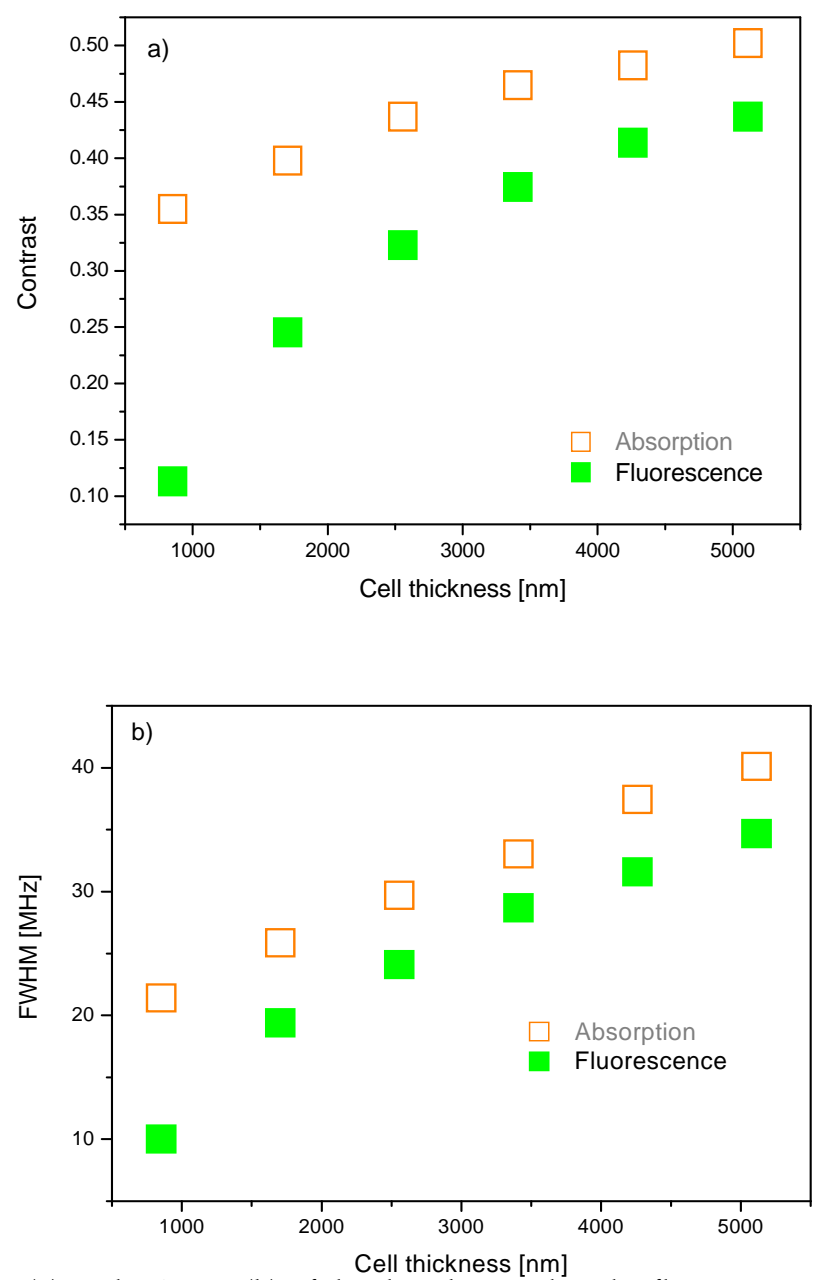

Fig. 22. Contrast (a) and FWHM (b) of the dip observed in the fluorescence and absorption.

\section{Conclusion}

The application of the nanometric cell provides a new opportunity for significant enhancement of the resolution in laser spectroscopy of thermal cell, without application of complex atomic beam or laser cooling systems. This simple approach makes it possible to study also the dynamic processes in the absorption and fluorescence. It is shown that when the Cs vapor layer thickness is about the wavelength of the irradiating light, the fluorescence spectrum is significantly narrower than the absorption one and the absorption coefficient does not grow linearly with the thickness of the atomic layer. The observed in the optical domain coherent Dicke narrowing of atomic transitions is strongest at $L=\lambda / 2$, while further expanding of vapor layer thickness results in periodical collapse and revival of this coherent effect. Demonstrated and analyzed is the significant difference between the 
saturation of open and closed transitions under conditions of very well expressed Dicke revival $(\mathrm{L}=1.5 \lambda)$. The appearance of a narrow structure in the sub-Doppler fluorescence profile is studied both experimentally and theoretically, distinguishing the difference between open and closed transitions. Theoretical simulations have predicted a significant enhancement of the amplitude of the narrow dip observed in the fluorescence profile with the ETC thickness enlargement. The prediction is confirmed experimentally, demonstrating that the narrow structure in the fluorescence at $L=6 \lambda$ has a very good potential for development of frequency references.

\section{Acknowledgement}

This chapter is dedicated to the memory of our colleague and friend Prof. Solomon Saltiel, great teacher in linear and non-linear spectroscopy.

The work is partially supported by the Bulgarian Fund for Scientific Research (grant No: DO 02-108/22.05.2009 and grant DMU 02/17 - 18.12.2009) and Indian -Bulgarian (BIn-2/07) bilateral contract.

\section{References}

Andreeva, C., Atvars, A., Auzinsh, M., Bluss, K., Cartaleva, S., Petrov, L. \& Slavov, D. (2007, a) Ground-state magneto-optical resonances in Cesium vapour confined in an extremely thin cell, Physical Review A, Vol.76, 063804.

Andreeva, C., Cartaleva, S., Dancheva, Y., Biancalana, V., Burchianti, A., Marinelli, C., Mariotti, E., Moi, L. \& Nasyrov, K. (2002) Coherent spectroscopy of degenerate two-level systems in Cs, Physical Review A, Vol.66, 012502.

Andreeva, C., Cartaleva, S., Petrov, L., Saltiel, S.M., Sarkisyan, D., Varzhapetyan, T., Bloch, D. \& Ducloy, M. (2007, b) Saturation effects in the sub-Doppler spectroscopy of Cesium vapor confined in an extremely thin cell, Physical Review A, Vol.76, 013837.

Briaudeau, S., Bloch, D. \& Ducloy, M. (1996) Detection of slow atoms in laser spectroscopy of a thin vapor film, Europhysics Letters, Vol.35, pp. 337-342

Briaudeau, S., Saltiel, S., Nihenius, G., Bloch, D. \& Ducloy, M. (1998) Coherent Doppler narrowing in a thin vapor cell: Observation of the Dicke regime in optical domain, Physical Review A, Vol.57, pp. R3169-R3172

Cartaleva, S., Saltiel, S., Sargsyan, A., Sarkisyan, D., Slavov, D., Todorov, P., \& Vaseva, K. (2009) Sub-Doppler spectroscopy of cesium vapor layers with nanometric and micrometric thickness, Journal of the Optical Society of America B: Optical Physics, Vol.26, pp.1999-2006.

Cartaleva, S., Sargsyan, A., Sarkisyan, D., Slavov, D. \& Vaseva, K. (2011) New narrow resonance in the fluorescence of closed optical transition observed in nanometric Cs - vapor layers, Proceedings of SPIE - The International Society for Optical Engineering, Vol.7747, 77470H

Dutier, G., Saltiel, S., Bloch D. \& Ducloy M. (2003, a) Revisiting optical spectroscopy in a thin vapor cell: mixing of reflection and transmission as a Fabri-Perot microcavity effect, Journal of the Optical Society of America B: Optical Physics, Vol.20, 793-800. 
Dutier, G., Yarovitski, A., Saltiel, S., Papoyan, A., Sarkisyan, D., Bloch, D. \& Ducloy, M. $(2003, b)$ Collapse and revival of a Dicke-type coherent narrowing in a sub-micron thick vapor cell transmission spectroscopy, Europhysics Letters, Vol.63, pp.35-41.

Maurin, I., Todorov, P., Hamdi, I., Yarovitski, A., Dutier, G., Sarkisyan, D., Saltiel, S., Gorza, M.-P., Fichet, M., Bloch, D. \& Ducloy, M. (2005) Probing an atomic gas confined in a nanocell, Journal of Physics: Conference Series, Vol.19, pp.20-29.

Romer, R. \& Dicke, R. (1955)New technique for high-resolution microwave spectroscopy, Physical Review, Vol.99, pp.532-536.

Sargsyan, A., Hakhumyan, G., Papoyan, A., Sarkisyan, D., Atvars, A., \& Auzinsh, M. (2008, a) A novel approach to quantitative spectroscopy of atoms in a magnetic field and applications based on an atomic vapor cell with $\mathrm{L}=\lambda$, Applied Physics Letters, Vol.93, 021119

Sargsyan, A., Sarkisyan, D., Papoyan, A., Pashayan-Leroy, Y., Moroshkin, P., Weis, A., Khanbekyan, A., Mariotti, E. \& Moi, L. (2008, b) Saturated absorption spectroscopy: elimination of crossover resonances by use of a nanocell, Laser Physics, Vol.18, 749-755.

Sarkisyan, D., Bloch, D., Papoyan, A. \& Ducloy, M. (2001) Sub-Doppler spectroscopy by sub micron thin Cs vapour layer, Optics Communications, Vol. 200, pp.201-208.

Sarkisyan, D., Varzhapetyan, T., Sarkisyan, A., Malakyan, Yu., Papoyan, A., Lezama, A., Bloch, D. \& Ducloy, M. (2004) Spectroscopy in an extremely thin cell: Comparing the cell-length dependence in fluorescence and in absorption techniques, Physical Review A, Vol.69, 065802.

Todorov, P., Vaseva, K., Cartaleva, S., Slavov, D., Maurin, I. \& Saltiel, S. (2008) Absorption and fluorescence in saturation regime of Cs-vapour layer with thickness close to the light wavelength, Proceedings of SPIE - The International Society for Optical Engineering, Vol.7027, 7027R

Varzhapetyan, T., Sarkisyan, D., Petrov, L., Andreeva, C., Slavov, D., Saltiel, S., Markovski, A., Todorov, G. \& Cartaleva, S. (2005) Sub-Doppler spectroscopy and coherence resonances in submicron Cs vapour layer, Proceedings of SPIE - The International Society for Optical Engineering, Vol.5830, pp.196-200.

Vaseva, K., Slavov, D., Todorov, P., Taslakov, M., Saltiel, S. \& Cartaleva, S. (2011) High resolution spectroscopy of Cesium-vapor layer with micrometric thickness for development of frequency reference, submitted for publication 




\author{
Advanced Photonic Sciences \\ Edited by Dr. Mohamed Fadhali
}

ISBN 978-953-51-0153-6

Hard cover, 374 pages

Publisher InTech

Published online 21, March, 2012

Published in print edition March, 2012

The new emerging field of photonics has significantly attracted the interest of many societies, professionals and researchers around the world. The great importance of this field is due to its applicability and possible utilization in almost all scientific and industrial areas. This book presents some advanced research topics in photonics. It consists of 16 chapters organized into three sections: Integrated Photonics, Photonic Materials and Photonic Applications. It can be said that this book is a good contribution for paving the way for further innovations in photonic technology. The chapters have been written and reviewed by well-experienced researchers in their fields. In their contributions they demonstrated the most profound knowledge and expertise for interested individuals in this expanding field. The book will be a good reference for experienced professionals, academics and researchers as well as young researchers only starting their carrier in this field.

\title{
How to reference
}

In order to correctly reference this scholarly work, feel free to copy and paste the following:

Stefka Cartaleva, Anna Krasteva, Armen Sargsyan, David Sarkisyan, Dimitar Slavov, Petko Todorov and Kapka Vaseva (2012). High Resolution Laser Spectroscopy of Cesium Vapor Layers with Nanometric Thickness, Advanced Photonic Sciences, Dr. Mohamed Fadhali (Ed.), ISBN: 978-953-51-0153-6, InTech, Available from: http://www.intechopen.com/books/advanced-photonic-sciences/high-resolution-laserspectroscopy-of-alkali-vapor-layers-with-nanometric-thickness

\section{INTECH}

open science | open minds

\section{InTech Europe}

University Campus STeP Ri

Slavka Krautzeka 83/A

51000 Rijeka, Croatia

Phone: +385 (51) 770447

Fax: +385 (51) 686166

www.intechopen.com

\section{InTech China}

Unit 405, Office Block, Hotel Equatorial Shanghai

No.65, Yan An Road (West), Shanghai, 200040, China

中国上海市延安西路65号上海国际贵都大饭店办公楼 405 单元

Phone: +86-21-62489820

Fax: $+86-21-62489821$ 
(C) 2012 The Author(s). Licensee IntechOpen. This is an open access article distributed under the terms of the Creative Commons Attribution 3.0 License, which permits unrestricted use, distribution, and reproduction in any medium, provided the original work is properly cited. 